\title{
Viscosity solution of system of integro-partial differential equations with interconnected obstacles of non-local type without Monotonicity Conditions
}

\author{
Said Hamadène*, Mohamed Mnif ${ }^{\dagger}$ and Sarra Neffati..$^{\ddagger \S}$
}

May 15, 2019

\begin{abstract}
In this paper, we study a system of second order integro-partial differential equations with interconnected obstacles with non-local terms, related to an optimal switching problem with the jump-diffusion model. Getting rid of the monotonicity condition on the generators with respect to the jump component, we construct a continuous viscosity solution which is unique in the class of functions with polynomial growth. In our study, the main tool is the notion of reflected backward stochastic differential equations with jumps with interconnected obstacles for which we show the existence of a solution.
\end{abstract}

Keywords: Integro-partial differential equations, Interconnected obstacles, Non-local terms, Viscosity solution, Switching problem, Reflected backward stochastic differential equations with jumps.

\section{Introduction}

Let us consider the following system of integro-partial differential equations (IPDEs for short) with interconnected obstacles with non-local terms: $\forall i \in \mathcal{I}:=\{1, \ldots, m\}$,

$$
\left\{\begin{array}{l}
\min \left\{u^{i}(t, x)-\max _{j \in \mathcal{I}^{-i}}\left(u^{j}(t, x)-g_{i j}(t, x)\right) ;-\partial_{t} u^{i}(t, x)-\mathcal{L} u^{i}(t, x)-K u^{i}(t, x)\right. \\
\left.\quad-\bar{f}_{i}\left(t, x,\left(u^{k}(t, x)\right)_{k=1, m},\left(\sigma^{T} D_{x} u^{i}\right)(t, x), B_{i} u^{i}(t, x)\right)\right\}=0, \quad(t, x) \in[0, T] \times \mathbb{R}^{k} ; \\
u^{i}(T, x)=h_{i}(x),
\end{array}\right.
$$

where $\mathcal{I}^{-i}:=\mathcal{I}-\{i\}$ for any $i \in \mathcal{I}$ and the operators $\mathcal{L}, K$ and $B_{i}$ are defined as follows:

$$
\begin{aligned}
\mathcal{L} u^{i}(t, x) & :=b(t, x)^{\top} D_{x} u^{i}(t, x)+\frac{1}{2} \operatorname{Tr}\left[\left(\sigma \sigma^{\top}\right)(t, x) D_{x x}^{2} u^{i}(t, x)\right] \\
K u^{i}(t, x) & :=\int_{E}\left(u^{i}(t, x+\beta(x, e))-u^{i}(t, x)-\beta(x, e)^{\top} D_{x} u^{i}(t, x)\right) \lambda(d e) \text { and } \\
B_{i} u^{i}(t, x) & :=\int_{E} \gamma_{i}(x, e)\left(u^{i}(t, x+\beta(x, e))-u^{i}(t, x)\right) \lambda(d e) .
\end{aligned}
$$

\footnotetext{
${ }^{*}$ LMM, Le Mans Université, Avenue Olivier Messiaen, 72085 Le Mans, Cedex 9, France. e-mail: hamadene@univ-lemans.fr;

${ }^{\dagger}$ University of Tunis El Manar, Laboratoire de Modélisation Mathématique et Numérique e-mail: mohamed.mnif@enit.rnu.tn;

${ }^{\ddagger}$ University of Tunis El Manar, Ecole Nationale d’Ingénieurs de Tunis, Laboratoire de Modélisation Mathématique et Numérique, 1002, Tunis, Tunisie;

${ }^{\S}$ LMM, Le Mans Université, Avenue Olivier Messiaen, 72085 Le Mans, Cedex 9, France. e-mail: sarra.neffatidenit.utm.tn
} 
In the above, $D_{x} u^{i}$ and $D_{x x}^{2} u^{i}$ are the gradient and Hessian matrix of $u^{i}$ with respect to its second variable $x$, respectively; (.) $)^{\top}$ is the transpose and $\lambda$ is a finite Lévy measure on $E:=\mathbb{R}^{l}-\{0\}$.

As pointed out previously, in (1.1) the operators $K u^{i}$ and $B_{i} u^{i}$ at $(t, x)$ involve the values of $u^{i}$ in the whole space $\mathbb{R}^{k}$ and not only locally which means that the system (1.1) is of non-local type. On the other hand, note that, the IPDEs (1.1) have one reflecting obstacle which depends on the solution $\left(u^{i}\right)_{i=1, m}$.

A special case of this type of system of IPDEs with interconnected obstacles occurs in the context of optimal switching control problems when the dynamics of the state variables are described by a Lévy process $\left(X_{s}^{t, x}\right)_{s \leq T}$ solving the following stochastic differential equation:

$$
\left\{\begin{array}{l}
d X_{s}^{t, x}=b\left(s, X_{s}^{t, x}\right) d s+\sigma\left(s, X_{s}^{t, x}\right) d B_{s}+\int_{E} \beta\left(X_{s^{-}}^{t, x}, e\right) \tilde{\mu}(d s, d e), \quad s \in[t, T] \\
X_{s}^{t, x}=x \in \mathbb{R}^{k}, \quad s \leq t
\end{array}\right.
$$

where $B:=\left(B_{s}\right)_{s \leq T}$ is a d-dimensional Brownian motion, $\mu$ an independent Poisson random measure with compensator $d s \lambda(d e)$ and $\tilde{\mu}(d s, d e):=\mu(d s, d e)-d s \lambda(d e)$ its compensated random measure.

In this setting, if for any $i \in \mathcal{I}, \bar{f}_{i}$ does not depend on $\left(u^{k}\right)_{k=1, m}, D_{x} u^{i}$ and $B_{i} u^{i}$ (see, e.g., [13]), the IPDEs (1.1) reduce to the Hamilton-Jacobi-Bellman system associated with the switching control problem whose value function is defined by

$$
u^{i}(t, x)=\sup _{\delta:=\left(\theta_{k}, \alpha_{k}\right)_{k \geq 0}} \mathbb{E}\left[\int_{t}^{T} \bar{f}^{\delta}\left(s, X_{s}^{t, x}\right) d s-\sum_{k \geq 1} g_{\alpha_{k-1} \alpha_{k}}\left(\theta_{k}, X_{\theta_{k}}^{t, x}\right) \mathbf{1}_{\left\{\theta_{k} \leq s\right\}}+h^{\delta}\left(X_{T}^{t, x}\right)\right],
$$

where :

(a) $\delta:=\left(\theta_{k}, \alpha_{k}\right)_{k \geq 0}$ is a strategy of switching in which $\left(\theta_{k}\right)_{k \geq 0}$ is an increasing sequence of stopping times and $\left(\alpha_{k}\right)_{k \geq 0}$ is a sequence of random variables with values in $\{1, \ldots, m\}$;

(b) $\bar{f}^{\delta}\left(s, X_{s}^{t, x}\right)$ is the instantaneous payoff when run under $\delta$ and $h^{\delta}\left(X_{T}^{t, x}\right)$ is the terminal payoff ;

(c) $g_{i j}$ is the switching cost function when moving from mode $i$ to mode $j(i, j \in \mathcal{I}, i \neq j)$.

We mention that the system of IPDEs (1.1) and related to optimal switching problems of jump-diffusion process have been studied in [5], [16], [13], [14] or [17].

An alternative method to tackle system (1.1), is to use the following system of reflected backward stochastic differential equations (RBSDEs for short) with jumps with interconnected obstacles (or oblique reflection): $\forall i \in \mathcal{I}$ and $s \in[t, T]$,

$$
\left\{\begin{aligned}
& Y_{s}^{i, t, x}= h_{i}\left(X_{T}^{t, x}\right)+\int_{s}^{T} \bar{f}_{i}\left(r, X_{r}^{t, x},\left(Y_{r}^{k, t, x}\right)_{k \in \mathcal{I}}, Z_{r}^{i, t, x}, \int_{E} V_{r}^{i, t, x}(e) \gamma_{i}\left(X_{r}^{t, x}, e\right) \lambda(d e)\right) d r \\
& \quad+K_{T}^{i, t, x}-K_{s}^{i, t, x}-\int_{s}^{T} Z_{r}^{i, t, x} d B_{r}-\int_{s}^{T} \int_{E} V_{r}^{i, t, x}(e) \tilde{\mu}(d r, d e), \\
& Y_{s}^{i, t, x} \geqslant \max _{j \in \mathcal{I}^{-i}}\left(Y_{s}^{j, t, x}-g_{i j}\left(s, X_{s}^{t, x}\right)\right), \\
& \int_{0}^{T}\left(Y_{s}^{i, t, x}-\max _{j \in \mathcal{I}^{-i}}\left(Y_{s}^{j, t, x}-g_{i j}\left(s, X_{s}^{t, x}\right)\right)\right) d K_{s}^{i, t, x}=0 .
\end{aligned}\right.
$$

Note that, without the jump process, the system of RBSDEs with oblique reflection has been investigated in several papers including ([7, 12, 9, 15], etc.). However, with the presence of the process with jump, HamadèneZhao in [14], have proved that, if for any $i \in \mathcal{I}$, 
(i) $\gamma_{i} \geqslant 0$;

(ii) $\bar{f}_{i}\left(t, x,\left(y_{k}\right)_{k=1, m}, z, q\right)$ is non-decreasing with respect to $q$,

then system (1.5) has a unique solution $\left(Y^{i, t, x}, Z^{i, t, x}, V^{i, t, x}, K^{i, t, x}\right)_{i \in \mathcal{I}}$. Moreover, they have made the connection between this RBSDEs with the IPDEs (1.1) and they have shown the existence and uniqueness of the solution of (1.1), and more important a result of comparison. More precisely, under the conditions (i)-(ii) and due to the Markovian framework of randomness which stems from the jump-diffusion process $X^{t, x}$ in (1.3), then system (1.1) has a unique viscosity solution $\left(u^{i}\right)_{i \in \mathcal{I}}$ in the class of continuous functions with polynomial growth and which is defined by means of the representation of Feynman-Kac's type of the process $\left(Y^{i, t, x}\right)_{i \in \mathcal{I}}$, i.e.,

$$
\forall i \in \mathcal{I}, u^{i}(t, x)=Y_{t}^{i, t, x},(t, x) \in[0, T] \times \mathbb{R}^{k} .
$$

Conditions (i)-(ii), which will be referred as the monotonicity conditions, are needed in order to have the comparison result and to treat the problem roed by the operator $B_{i} u^{i}$ which is not well-defined for any arbitrary $u^{i}$.

Therefore, without assuming the above monotonicity conditions neither on $\gamma_{i}$ nor on $\bar{f}_{i}, i=1, \ldots, m$, the problem of existence and uniqueness of the viscosity solution of system 1.1 remains open. In this paper, we show that system (1.1) has a continuous viscosity solution which is unique in the class of functions with polynomial growth. As a by-product, we show also that, without the monotonicity conditions, the RBSDEs with jumps with interconnected obstacles (1.5) has a solution. Our method relies mainly on the characterization of the jump part of the RBSDEs (1.5) by means of the function $\left(u^{i}\right)_{i=1, m}$ defined in (1.6) and the jump-diffusion process $X^{t, x}$, when the measure $\lambda$ is finite.

The paper is organized as follows. In Section 2, we provide all the necessary notations and assumptions concerning the study of IPDEs (1.1) and related RBSDEs with jumps as well. In Section 3, we study the existence of a solution for system of RBSDEs with jumps (1.5) and Feynman-Kac representation (1.6). We show in Section 4 that the function $\left(u^{i}\right)_{i=1, m}$ is the unique viscosity solution of (1.1) in the class of continuous functions with polynomial growth. In the Appendix, we give another definition of the viscosity solution of system (1.1) which is inspired by the work by Hamadène-Zhao in [14].

\section{Preliminaries and notations}

Let $T>0$ be a given time horizon and $\left(\Omega, \mathcal{F}, \mathbb{F}:=\left(\mathcal{F}_{t}\right)_{t \leq T}, \mathbb{P}\right)$ be a stochastic basis such that $\mathcal{F}_{0}$ contains all the $P$-null sets of $\mathcal{F}, \mathcal{F}_{t^{+}}=\cap_{\varepsilon>0} \mathcal{F}_{t+\varepsilon}=\mathcal{F}_{t}$, and we suppose that the filtration is generated by the two following mutually independent processes :

(i) a d-dimensional standard Brownian motion $B:=\left(B_{t}\right)_{0 \leq t \leq T}$ and

(ii) a Poisson random measure $\mu$ on $\mathbb{R}^{+} \times E$, where $E:=\mathbb{R}^{l}-\{0\}$ is equipped with its Borel field $\mathbb{B}(E),(l \geqslant$ 1 fixed). Let $\nu(d t, d e)=d t \lambda(d e)$ be its compensated process such that $\{\tilde{\mu}([0, t] \times A)=(\mu-\nu)([0, t] \times$ $A)\}_{t \leq T}$ is a martingale for every $A \in \mathcal{B}(E)$ satisfying $\lambda(A)<\infty$. The measure $\lambda$ is assumed to be $\sigma$-finite on $(E, \mathcal{B}(E))$ and integrates the function $\left(1 \wedge|e|^{2}\right)_{e \in E}$.

Let us introduce the following spaces: 
a) $\mathcal{P}$ (resp. P) is the $\sigma$-algebra of $\mathbb{F}$-progressively measurable (resp. $\mathbb{F}$-predictable) sets on $\Omega \times[0, T]$;

b) $\mathcal{L}^{2}(\lambda)$ is the space of Borel measurable functions $(\varphi(e))_{e \in E}$ from $E$ into $\mathbb{R}$ such that $\int_{E}|\varphi(e)|^{2} \lambda(d e)<$ $\infty$;

c) $\mathcal{S}^{2}$ is the space of RCLL (right continuous with left limits) $\mathcal{P}$-measurable and $\mathbb{R}$-valued processes $Y:=$ $\left(Y_{s}\right)_{s \leq T}$ such that $\mathbb{E}\left[\sup _{0 \leq t \leq T}\left|Y_{s}\right|^{2}\right]<\infty$

d) $\mathcal{A}^{2}$ is the subspace of $\mathcal{S}^{2}$ of continuous non-decreasing processes $K:=\left(K_{t}\right)_{t \leq T}$ such that $K_{0}=0$;

e) $\mathcal{H}^{2, d}$ is the space of $\mathcal{P}$-measurable and $\mathbb{R}^{d}$-valued processes $Z:=\left(Z_{s}\right)_{s \leq T}$ such that $\mathbb{E}\left[\int_{0}^{T}\left|Z_{s}\right|^{2} d s\right]<$ $\infty$;

f) $\mathcal{H}^{2}\left(\mathcal{L}^{2}(\lambda)\right)$ is the space of $\mathbf{P}$-measurable and $\mathcal{L}^{2}(\lambda)$-valued processes $U:=\left(U_{s}\right)_{s \leq T}$ such that $\mathbb{E}\left[\int_{0}^{T} \int_{E}\left|U_{s}(e)\right|^{2} \lambda(d e) d s\right]<\infty$.

For a RCLL process $\left(\theta_{s}\right)_{s \leq T}$, we define for any $s \in(0, T], \theta_{s^{-}}=\lim _{r} \nearrow_{s} \theta_{r}$ and $\Delta_{s} \theta=\theta_{s}-\theta_{s^{-}}$is the jump size of $\theta$ at $s$.

Now, for any $(t, x) \in[0, T] \times \mathbb{R}^{k}$, let $\left(X_{s}^{t, x}\right)_{s \leq T}$ be the stochastic process solution of the following stochastic differential equation (SDE for short) of diffusion-jump type:

$$
\left\{\begin{array}{l}
d X_{s}^{t, x}=b\left(s, X_{s}^{t, x}\right) d s+\sigma\left(s, X_{s}^{t, x}\right) d B_{s}+\int_{E} \beta\left(X_{s^{-}}^{t, x}, e\right) \tilde{\mu}(d s, d e), \quad s \in[t, T] \\
X_{s}^{t, x}=x \in \mathbb{R}^{k}, \quad 0 \leq s \leq t
\end{array}\right.
$$

where $b:[0, T] \times \mathbb{R}^{k} \rightarrow \mathbb{R}^{k}$ and $\sigma:[0, T] \times \mathbb{R}^{k} \rightarrow \mathbb{R}^{k \times d}$ are two continuous functions in $(t, x)$ and Lipschitz w.r.t $x$, i.e., there exists a positive constant $C$ such that

$$
\left|b(t, x)-b\left(t, x^{\prime}\right)\right|+\left|\sigma(t, x)-\sigma\left(t, x^{\prime}\right)\right| \leq C\left|x-x^{\prime}\right|, \quad \forall\left(t, x, x^{\prime}\right) \in[0, T] \times \mathbb{R}^{k+k} .
$$

Note that the continuity of $b, \sigma$ and (2.2) imply the existence of a constant $C$ such that

$$
|b(t, x)|+|\sigma(t, x)| \leq C(1+|x|), \quad \forall(t, x) \in[0, T] \times \mathbb{R}^{k} .
$$

Next, let $\beta: \mathbb{R}^{k} \times E \rightarrow \mathbb{R}^{k}$ be a measurable function such that for some real constant $c$,

$$
|\beta(x, e)| \leq c(1 \wedge|e|) \quad \text { and } \quad\left|\beta(x, e)-\beta\left(x^{\prime}, e\right)\right| \leq c\left|x-x^{\prime}\right|(1 \wedge|e|), \quad \forall e \in E \text { and } x, x^{\prime} \in \mathbb{R}^{k} \text {. }
$$

Conditions (2.2), (2.3) and (2.4) ensure, for any $(t, x) \in[0, T] \times \mathbb{R}^{k}$, the existence and uniqueness of a solution of equation (2.1) (see [8] for more details). Moreover, it satisfies the following estimate: $\forall p \geqslant 1$,

$$
\mathbb{E}\left[\sup _{s \leq T}\left|X_{s}^{t, x}\right|^{p}\right] \leqslant C\left(1+|x|^{p}\right) .
$$

Next, let us introduce the following deterministic functions $\left(\bar{f}_{i}\right)_{i=1, \ldots, m},\left(h_{i}\right)_{i=1, \ldots, m}$ and $\left(g_{i j}\right)_{i, j=1, \ldots, m}$ defined as follows : for any $i, j \in\{1, \ldots, m\}$,

a) $\bar{f}_{i}:(t, x, \vec{y}, z, q) \in[0, T] \times \mathbb{R}^{k+m+d+1} \longmapsto \bar{f}_{i}(t, x, \vec{y}, z, q) \in \mathbb{R}\left(\vec{y}:=\left(y^{1}, \ldots, y^{m}\right)\right) ;$

b) $g_{i j}:(t, x) \in[0, T] \times \mathbb{R}^{k} \longmapsto g_{i j}(t, x) \in \mathbb{R}$;

c) $h_{i}: x \in \mathbb{R}^{k} \longmapsto h_{i}(x) \in \mathbb{R}$.

Additionally we assume that they satisfy: 
(H1) For any $i \in\{1, \ldots, m\}$,

(i) The function $(t, x) \mapsto \bar{f}_{i}(t, x, \vec{y}, z, q)$ is continuous, uniformly w.r.t. the variables $(\vec{y}, z, q)$,

(ii) The function $\bar{f}_{i}$ is Lipschitz continuous w.r.t. the variables $(\vec{y}, z, q)$ uniformly in $(t, x)$, i.e., there exists a positive constant $C_{i}$ such that for any $(t, x) \in[0, T] \times \mathbb{R}^{k},(\vec{y}, z, q)$ and $\left(\vec{y}_{1}, z_{1}, q_{1}\right)$ elements of $\mathbb{R}^{m+d+1}$ :

$$
\left|\bar{f}_{i}(t, x, \vec{y}, z, q)-\bar{f}_{i}\left(t, x, \vec{y}_{1}, z_{1}, q_{1}\right)\right| \leq C_{i}\left(\left|\vec{y}-\vec{y}_{1}\right|+\left|z-z_{1}\right|+\left|q-q_{1}\right|\right)
$$

(iii) The mapping $(t, x) \mapsto \bar{f}_{i}(t, x, 0,0,0)$ has polynomial growth in $x$, i.e., there exist two constants $C>0$ and $p \geqslant 1$ such that for any $(t, x) \in[0, T] \times \mathbb{R}^{k}$,

$$
\left|\bar{f}_{i}(t, x, 0,0,0)\right| \leq C\left(1+|x|^{p}\right) .
$$

(iv) For any $i \in \mathcal{I}$ and $j \in \mathcal{I}^{-i}$, the mapping $y^{j} \mapsto \bar{f}_{i}\left(t, x, y^{1}, \ldots, y^{j-1}, y^{j}, y^{j+1}, \ldots, y^{m}, z, q\right)$ is nondecreasing whenever the other components $\left(t, x, y^{1}, \ldots, y^{j-1}, y^{j+1}, \ldots, y^{m}, z, q\right)$ are fixed.

Next, for any $i \in\{1, \ldots, m\}$, let $\gamma_{i}: \mathbb{R}^{k} \times E \rightarrow \mathbb{R}$ be a $\mathbb{B}\left(\mathbb{R}^{k}\right) \otimes \mathbb{B}(E)$-measurable functions such that for some constant $C>0$ :

$$
\left|\gamma_{i}(x, e)\right| \leq C(1 \wedge|e|), \quad \forall(x, e) \in \mathbb{R}^{k} \times E .
$$

Finally let us define the function $\left(f_{i}\right)_{i=1, \ldots, m}$ on $[0, T] \times \mathbb{R}^{k+m+d} \times \mathcal{L}^{2}(\lambda)$, as follows:

$$
f_{i}(t, x, \vec{y}, z, v):=\bar{f}_{i}\left(t, x, \vec{y}, z, \int_{E} v(e) \gamma_{i}(x, e) \lambda(d e)\right) .
$$

Note that since $\bar{f}_{i}$ is uniformly Lipschitz in $(\vec{y}, z, q)$ and $\gamma_{i}$ verifies (2.8) then the function $f_{i}$ enjoy the two following properties:

(a) $f_{i}$ is Lipschitz continuous w.r.t. the variables $(\vec{y}, z, v)$ uniformly in $(t, x)$,

(b) The mapping $(t, x) \mapsto f_{i}(t, x, 0,0,0)$ is continuous with polynomial growth.

(H2) $\forall i, j \in\{1, \ldots, m\}, g_{i i}=0$ and for $i \neq j, g_{i j}(t, x)$ is non-negative, jointly continuous in $(t, x)$ with polynomial growth and satisfies the following non free loop property :

For any $(t, x) \in[0, T] \times \mathbb{R}^{k}$, for any sequence of indices $i_{1}, \ldots, i_{k}$ such that $i_{1}=i_{k}$ and $\operatorname{card}\left\{i_{1}, \ldots, i_{k}\right\}=$ $k-1(k \geq 3)$ we have

$$
g_{i_{1} i_{2}}(t, x)+g_{i_{2} i_{3}}(t, x)+\ldots+g_{i_{k} i_{1}}(t, x)>0 .
$$

(H3) For $i \in\{1, \ldots, m\}$, the function $h_{i}$, which stands for the terminal condition, is continuous with polynomial growth and satisfies the following consistency condition:

$$
\forall x \in \mathbb{R}^{k}, h_{i}(x) \geqslant \max _{j \in \mathcal{I}^{-i}}\left(h_{j}(x)-g_{i j}(T, x)\right) .
$$

(H4)-(i) $\forall i \in \mathcal{I}, \gamma_{i} \geqslant 0$;

(H4)-(ii) The mapping $q \in \mathbb{R} \longmapsto \bar{f}_{i}(t, x, \vec{y}, z, q)$ is non-decreasing when the other components $(t, x, \vec{y}, z)$ are fixed. 
The main objective of this paper is to study the following system of integro-partial differential equations (IPDEs) with interconnected obstacles: for any $i \in \mathcal{I}:=\{1, \ldots, m\}$,

$$
\left\{\begin{array}{l}
\min \left\{u^{i}(t, x)-\max _{j \in \mathcal{I}^{-i}}\left(u^{j}(t, x)-g_{i j}(t, x)\right) ;-\partial_{t} u^{i}(t, x)-\mathcal{L} u^{i}(t, x)-K u^{i}(t, x)\right. \\
\left.\quad-\bar{f}_{i}\left(t, x,\left(u^{k}(t, x)\right)_{k=1, m},\left(\sigma^{T} D_{x} u^{i}\right)(t, x), B_{i} u^{i}(t, x)\right)\right\}=0 \\
u^{i}(T, x)=h_{i}(x)
\end{array}\right.
$$

where $\mathcal{L}$ is the second-order local operator

$$
\mathcal{L} \varphi(t, x):=b(t, x)^{\top} D_{x} \varphi(t, x)+\frac{1}{2} \operatorname{Tr}\left[\left(\sigma \sigma^{\top}\right)(t, x) D_{x x}^{2} \varphi(t, x)\right] ;
$$

and the two non-local operators $K$ and $B_{i}$ are defined as follows

$$
\begin{aligned}
& K \varphi(t, x):=\int_{E}\left(\varphi(t, x+\beta(x, e))-\varphi(t, x)-\beta(x, e)^{\top} D_{x} \varphi(t, x)\right) \lambda(d e) \text { and } \\
& B_{i} \varphi(t, x):=\int_{E} \gamma_{i}(x, e)(\varphi(t, x+\beta(x, e))-\varphi(t, x)) \lambda(d e) .
\end{aligned}
$$

for any $\mathbb{R}$-valued function $\varphi(t, x)$ such that $D_{x} \varphi(t, x)$ and $D_{x x}^{2} \varphi(t, x)$ are defined.

\section{Systems of Reflected BSDEs with Jumps with Oblique Reflection}

The system of IPDEs (2.12) is deeply related with the following system of reflected BSDEs with jumps with interconnected obstacles (or oblique reflection) associated with $\left(\left(\bar{f}_{i}\right)_{i \in \mathcal{I}},\left(g_{i j}\right)_{i, j \in \mathcal{I}},\left(h_{i}\right)_{i \in \mathcal{I}}\right)$ :

$\forall i=1, \ldots, m$ and $s \in[t, T]$,

$$
\left\{\begin{aligned}
& Y^{i, t, x} \in \mathcal{S}^{2}, Z^{i, t, x} \in \mathcal{H}^{2, d}, V^{i, t, x} \in \mathcal{H}^{2}\left(\mathcal{L}^{2}(\lambda)\right), \text { and } K^{i, t, x} \in \mathcal{A}^{2} ; \\
& Y_{s}^{i, t, x}= h_{i}\left(X_{T}^{t, x}\right)+\int_{s}^{T} \bar{f}_{i}\left(r, X_{r}^{t, x},\left(Y_{r}^{k, t, x}\right)_{k \in \mathcal{I}}, Z_{r}^{i, t, x}, \int_{E} V_{r}^{i, t, x}(e) \gamma_{i}\left(X_{r}^{t, x}, e\right) \lambda(d e)\right) d r \\
& \quad+K_{T}^{i, t, x}-K_{s}^{i, t, x}-\int_{s}^{T} Z_{r}^{i, t, x} d B_{r}-\int_{s}^{T} \int_{E} V_{r}^{i, t, x}(e) \tilde{\mu}(d r, d e), \\
& \begin{array}{rl}
Y_{s}^{i, t, x} \geqslant & \max _{j \in \mathcal{I}^{-i}}\left(Y_{s}^{j, t, x}-g_{i j}\left(s, X_{s}^{t, x}\right)\right),
\end{array} \\
& \int_{0}^{T}\left(Y_{s}^{i, t, x}-\max _{j \in \mathcal{I}^{-i}}\left(Y_{s}^{j, t, x}-g_{i j}\left(s, X_{s}^{t, x}\right)\right)\right) d K_{s}^{i, t, x}=0 .
\end{aligned}\right.
$$

This system of reflected BSDEs with jumps with interconnected obstacles (3.1) has been considered by Hamadène and Zhao in [14] where issues of existence and uniqueness of the solution, and the relationship between the solution of (3.1) and the one of system 2.12), are considered. Actually, it is shown:

Theorem 3.1 (see [14]).

Assume that the deterministic functions $\left(\bar{f}_{i}\right)_{i \in \mathcal{I}},\left(g_{i j}\right)_{i, j \in \mathcal{I}},\left(h_{i}\right)_{i \in \mathcal{I}}$ and $(\gamma)_{i \in \mathcal{I}}$ verify Assumptions (H1)-(H3) and (H4). Then, we have:

i) The system (3.1) has a unique solution $\left(Y^{i, t, x}, Z^{i, t, x}, V^{i, t, x}, K^{i, t, x}\right)_{i \in \mathcal{I}}$. 
ii) There exists a deterministic continuous functions $\left(u^{i}\right)_{i \in \mathcal{I}}$ of polynomial growth, defined on $[0, T] \times \mathbb{R}^{k}$, such that:

$$
\forall s \in[t, T], Y_{s}^{i, t, x}=u^{i}\left(s, X_{s}^{t, x}\right),
$$

In our setting, we also consider the system (3.1) without assuming Assumption (H4). We then have the following result.

Theorem 3.2 Assume that the functions $\left(\bar{f}_{i}\right)_{i \in \mathcal{I}},\left(g_{i j}\right)_{i, j \in \mathcal{I}}$ and $\left(h_{i}\right)_{i \in \mathcal{I}}$ verify Assumptions (H1)-(H3). Then the system (3.1) has a solution $\left(Y^{i, t, x}, Z^{i, t, x}, V^{i, t, x}, K^{i, t, x}\right)_{i \in \mathcal{I}}$.

Proof: The proof is divided into three steps.

\section{Step 1: The iterative construction}

For any $n \geq 0$, let $\left(\left(Y^{i, n}, Z^{i, n}, V^{i, n}, K^{i, n}\right)_{i \in \mathcal{I}}\right)_{n \geq 0}$ be the sequence of processes defined recursively as follows:

$$
\begin{gathered}
\left(Y^{i, 0}, Z^{i, 0}, V^{i, 0}, K^{i, 0}\right)=(0,0,0,0) \text { for all } i \in \mathcal{I} \text { and for } n \geq 1 \text { and } s \leq T, \\
\left\{\begin{array}{c}
Y^{i, n} \in \mathcal{S}^{2}, Z^{i, n} \in \mathcal{H}^{2, d}, V^{i, n} \in \mathcal{H}^{2}\left(\mathcal{L}^{2}(\lambda)\right), \text { and } K^{i, n} \in \mathcal{A}^{2} \\
Y_{s}^{i, n}=h_{i}\left(X_{T}^{t, x}\right)+\int_{s}^{T} \bar{f}_{i}\left(r, X_{s}^{t, x},\left(Y_{r}^{k, n}\right)_{k \in \mathcal{I}}, Z_{r}^{i, n}, \int_{E} V_{r}^{i, n-1}(e) \gamma_{i}\left(X_{r}^{t, x}, e\right) \lambda(d e)\right) d r \\
\quad+K_{T}^{i, n}-K_{s}^{i, n}-\int_{s}^{T} Z_{r}^{i, n} d B_{r}-\int_{s}^{T} \int_{E} V_{r}^{i, n}(e) \tilde{\mu}(d r, d e), \\
Y_{s}^{i, n} \geqslant \max _{j \in \mathcal{I}^{-i}}\left(Y_{s}^{j, n}-g_{i j}\left(s, X_{s}^{t, x}\right)\right), \\
\int_{0}^{T}\left(Y_{s}^{i, n}-\max _{j \in \mathcal{I}^{-i}}\left(Y_{s}^{j, n}-g_{i j}\left(s, X_{s}^{t, x}\right)\right)\right) d K_{s}^{i, n}=0 .
\end{array}\right.
\end{gathered}
$$

First we notice that by Theorem (3.1), the solution of this system (3.2) exists and is unique. More precisely: for any $i \in \mathcal{I}$, the generators $\bar{f}_{i}$ does not depend on $V^{i, n}$, noting that $V^{i, n-1}$ is already given and the functions $\left(h_{i}\right)_{i \in \mathcal{I}}$ and $\left(g_{i j}\right)_{i, j \in \mathcal{I}}$ satisfy the Assumptions (H1)-(H3) and (H4) as well. Next, since the setting is Markovian and using an induction argument on $n$, it follows that:

(a) there exists a deterministic continuous functions of polynomial growth $u^{i, n}:[0, T] \times \mathbb{R}^{k} \rightarrow \mathbb{R}$, such that for any $s \in[t, T], Y_{s}^{i, n}:=u^{i, n}\left(s, X_{s}^{t, x}\right)$,

(b) $V_{s}^{i, n}(e):=u^{i, n}\left(s, X_{s^{-}}^{t, x}+\beta\left(X_{s^{-}}^{t, x}, e\right)\right)-u^{i, n}\left(s, X_{s^{-}}^{t, x}\right), d s \otimes d \mathbb{P} \otimes d \lambda$ on $[t, T] \times \Omega \times E$.

Indeed, for $n=0$, the properties (a), (b) are valid. Assume now that they are satisfied for some $n-1$, with $n \geq 1$. Then $\left(Y^{i, n}, Z^{i, n}, V^{i, n}, K^{i, n}\right)$ verifies: for any $s \in[t, T]$ and $i \in \mathcal{I}$,

$$
\left\{\begin{array}{l}
Y_{s}^{i, n}=h_{i}\left(X_{T}^{t, x}\right)+\int_{s}^{T} \bar{f}_{i}\left(r, X_{r}^{t, x},\left(Y_{r}^{k, n}\right)_{k \in \mathcal{I}}, Z_{r}^{i, n}, \int_{E}\left\{u^{i, n-1}\left(r, X_{r^{-}}^{t, x}+\beta\left(X_{r^{-}}^{t, x}, e\right)\right)\right.\right. \\
\left.\left.\quad-u^{i, n-1}\left(r, X_{r^{-}}^{t, x}\right)\right\} \gamma_{i}\left(X_{r}^{t, x}, e\right) \lambda(d e)\right) d r+K_{T}^{i, n}-K_{s}^{i, n}-\int_{s}^{T} Z_{r}^{i, n} d B_{r}-\int_{s}^{T} \int_{E} V_{r}^{i, n}(e) \tilde{\mu}(d r, d e), \\
Y_{s}^{i, n} \geqslant \max _{j \in \mathcal{I}^{-i}}\left(Y_{s}^{j, n}-g_{i j}\left(s, X_{s}^{t, x}\right)\right), \\
\int_{0}^{T}\left(Y_{s}^{i, n}-\max _{j \in \mathcal{I}^{-i}}\left(Y_{s}^{j, n}-g_{i j}\left(s, X_{s}^{t, x}\right)\right)\right) d K_{s}^{i, n}=0 .
\end{array}\right.
$$


Hence, by Proposition 4.2 in [14], we deduce the existence of $u^{i, n}$ which is continuous and of polynomial growth. Finally as the measure $\lambda$ is finite, i.e., $\lambda(E)<\infty$, then we have the following relationship between the process $\left(V^{i, n}\right)_{i \in \mathcal{I}}$ and the deterministic functions $\left(u^{i, n}\right)_{i \in \mathcal{I}}$ (see [10], Proposition 3.3):

$$
V_{s}^{i, n}(e)=u^{i, n}\left(s, X_{s^{-}}^{t, x}+\beta\left(X_{s^{-}}^{t, x}, e\right)\right)-u^{i, n}\left(s, X_{s^{-}}^{t, x}\right), d s \otimes d \mathbb{P} \otimes d \lambda \text { on }[t, T] \times \Omega \times E .
$$

Thus, the two representations (a) and (b) hold true for any $n \geq 0$.

\section{Step 2: Switching representation}

In this step, we represent $Y^{i, n}$ as the value of an optimal switching problem. Indeed, let $\delta:=\left(\theta_{k}, \alpha_{k}\right)_{k \geq 0}$ be an admissible strategy of switching, i.e., $\left(\theta_{k}\right)_{k \geq 0}$ is an increasing sequence of stopping times with values in $[0, T]$ such that $\mathbb{P}\left[\theta_{k}<T, \forall k \geq 0\right]=0$ and $\forall k \geq 0, \alpha_{k}$ is a random variable $\mathcal{F}_{\theta_{k}}$-measurable with values in $\mathcal{I}$.

Next, with the admissible strategy $\delta:=\left(\theta_{k}, \alpha_{k}\right)_{k \geq 0}$ is associated a switching cost process $\left(A_{s}^{\delta}\right)_{s \leq T}$ defined by:

$$
A_{s}^{\delta}:=\sum_{k \geq 1} g_{\alpha_{k-1} \alpha_{k}}\left(\theta_{k}, X_{\theta_{k}}^{t, x}\right) \mathbf{1}_{\left\{\theta_{k} \leq s\right\}} \text { for } s<T, \text { and } A_{T}^{\delta}=\lim _{s \rightarrow T} A_{s}^{\delta} .
$$

Note that $\left(A_{s}^{\delta}\right)_{s \leq T}$ is an RCLL process. Now, for $s \leq T$, let us set $\eta_{s}:=\alpha_{0} \mathbf{1}_{\left\{\theta_{0\}}\right.}(s)+\sum_{k \geq 1} \alpha_{k} \mathbf{1}_{\left[\theta_{k} \leq s<\theta_{k+1}\right)}$ which stands for the indicator of the system at time $s$. Note that $\eta$ is in bijection with the strategy $\delta$. Finally, for any fixed $s \leq T$ and $i \in \mathcal{I}$, let us denote by $\mathcal{A}_{s}^{i}$ the following set of admissible strategies:

$$
\mathcal{A}_{s}^{i}:=\left\{\delta:=\left(\theta_{k}, \alpha_{k}\right)_{k \geq 0} \text { admissible strategy such that } \theta_{0}=s, \alpha_{0}=i \text { and } \mathbb{E}\left[\left(A_{T}^{\delta}\right)^{2}\right]<\infty\right\} .
$$

Now, let $\delta:=\left(\theta_{k}, \alpha_{k}\right)_{k \geq 0} \in \mathcal{A}_{s}^{i}$ and let us define the triplet of adapted processes $\left(P_{s}^{n, \delta}, N_{s}^{n, \delta}, Q_{s}^{n, \delta}\right)_{s \leq T}$ as follows: $\forall s \leq T$,

$$
\left\{\begin{array}{l}
P^{n, \delta} \text { is RCLL and } \mathbb{E}\left[\sup _{s \leq T}\left|P_{s}^{n, \delta}\right|^{2}\right]<\infty ; N^{n, \delta} \in \mathcal{H}^{2, d} \text { and } Q^{n, \delta} \in \mathcal{H}^{2}\left(\mathcal{L}^{2}(\lambda)\right) ; \\
P_{s}^{n, \delta}=h^{\delta}\left(X_{T}^{t, x}\right)-A_{T}^{\delta}+A_{s}^{\delta}+\int_{s}^{T} \bar{f}^{\delta}\left(r, X_{r}^{t, x}, P_{r}^{n, \delta}, N_{r}^{n, \delta}, \int_{E}\left\{u^{\delta, n-1}\left(r, X_{r^{-}}^{t, x}+\beta\left(X_{r^{-}}^{t, x}, e\right)\right)\right.\right. \\
\left.\left.\quad-u^{\delta, n-1}\left(r, X_{r^{-}}^{t, x}\right)\right\} \gamma^{\delta}\left(X_{r}^{t, x}, e\right) \lambda(d e)\right) d r-\int_{s}^{T} N_{n}^{\delta}(r) d B_{r}-\int_{s}^{T} \int_{E} Q_{n}^{\delta}(e)(r) \tilde{\mu}(d r, d e),
\end{array}\right.
$$

where for any $s \leq T$,

$$
h^{\delta}(x):=\sum_{k \geq 0} h_{\alpha_{k}}(x) \mathbf{1}_{\left[\theta_{k} \leq T<\theta_{k+1}\right)} \text { and } \bar{f}^{\delta}(s, x, \vec{y}, z, q):=\sum_{k \geq 0} \bar{f}_{\alpha_{k}}(r, x, \vec{y}, z, q) \mathbf{1}_{\left[\theta_{k} \leq r<\theta_{k+1}\right)} .
$$

Those series contain only a finite many terms as $\delta$ is admissible and then $\mathbb{P}\left[\theta_{n}<T, \forall n \geq 0\right]=0$. Note that, for any $(\bar{y}, \bar{z}, \bar{q}) \in \mathbb{R}^{1+d+1}, \bar{f}^{\delta}\left(s, X_{s}^{t, x}, \bar{y}, \bar{z}, \bar{q}\right)$ is equal to $\bar{f}^{\delta}\left(s, X_{s}^{t, x},\left(u^{k, n}\left(s, X_{s}^{t, x}\right)\right)_{k \in \mathcal{I}}, \bar{z}, \bar{q}\right)$. We mention that, in (3.5), the generators $\bar{f}^{\delta}$ does not depend on the variable $Q^{n, \delta} \in \mathcal{H}^{2}\left(\mathcal{L}^{2}(\lambda)\right)$.

Next, by a change of variable, the existence of $\left(P^{n, \delta}-A^{\delta}, N^{n, \delta}, Q^{n, \delta}\right)$ stems from the standard existence result of solutions of BSDEs with jumps by Tang-Li [19] since its generator $\bar{f}^{\delta}\left(s, X_{s}^{t, x}, ., z,.\right)$ is Lipschitz w.r.t $z$ and $A_{T}^{\delta}$ is square integrable. Furthermore, we have the following representation of $Y^{i, n}$ (see e.g. [13] for more details on this representation):

$$
Y_{s}^{i, n}=\operatorname{esssup}_{\delta \in \mathcal{A}_{s}^{i}}\left(P_{s}^{n, \delta}-A_{s}^{\delta}\right)=\left(P_{s}^{n, \delta^{*}}-A_{s}^{\delta^{*}}\right)
$$


for some $\delta^{*} \in \mathcal{A}_{s}^{i}$, which means that $\delta^{*}$ is an optimal strategy of the switching control problem.

\section{Step 3: Convergence result}

We now adapt the argument already used in [7, 10, 13] to justify a convergence result for the sequence $\left(Y^{i, n}, Z^{i, n}, V^{i, n}, K^{i, n}\right)_{n}$. For this, let us set: $\forall i \in \mathcal{I}$ and $n, p \geq 1$

$$
\begin{aligned}
& F_{i}\left(s, X_{s}^{t, x}, \vec{y}, z\right):= \\
& \quad \bar{f}_{i}\left(s, X_{s}^{t, x}, \vec{y}, z, \int_{E} V_{s}^{i, n-1}(e) \gamma_{i}\left(X_{s}^{t, x}, e\right) \lambda(d e)\right) \vee \bar{f}_{i}\left(s, X_{s}^{t, x}, \vec{y}, z, \int_{E} V_{s}^{i, p-1}(e) \gamma_{i}\left(X_{s}^{t, x}, e\right) \lambda(d e)\right),
\end{aligned}
$$

and let us consider the solution denoted by $\left(\hat{Y}^{i, n}, \hat{Z}^{i, n}, \hat{V}^{i, n}, \hat{K}^{i, n}\right)_{i \in \mathcal{I}}$ of the solution of the obliquely reflected BSDEs with jumps associated with $\left(\left(F_{i}\right)_{i \in \mathcal{I}},\left(g_{i j}\right)_{i, j \in \mathcal{I}},\left(h_{i}\right)_{i \in \mathcal{I}}\right)$. Moreover, once more, the following representation hold true: $\forall s \leq T$,

$$
\hat{Y}_{s}^{i, n}=\operatorname{esssup}_{\delta \in \mathcal{A}_{s}^{i}}\left(\hat{P}_{s}^{n, \delta}-A_{s}^{\delta}\right)=\left(\hat{P}_{s}^{n, \tilde{\delta}^{*}}-A_{s}^{\tilde{\delta}^{*}}\right),
$$

where $\left(\hat{P}^{n, \delta}, \hat{N}^{n, \delta}, \hat{Q}^{n, \delta}\right)$ is the solution of the BSDE (3.5) with generator $F^{\delta}(\ldots)$. Then by the comparison result (see Proposition 4.2 in [14]), between the solutions $Y^{i, n}$ and $\hat{Y}^{i, n}$, one deduce that

$$
Y_{s}^{i, n} \leq \hat{Y}_{s}^{i, n} \text { and } Y_{s}^{i, p} \leq \hat{Y}_{s}^{i, n}
$$

This combined with (3.7) and (3.8), leads to

$$
\left|Y_{s}^{i, n}-Y_{s}^{i, p}\right|^{2} \leq 2\left\{\left|\hat{P}_{s}^{n, \tilde{\delta}^{*}}-P_{s}^{n, \tilde{\delta}^{*}}\right|^{2}+\left|\hat{P}_{s}^{n, \tilde{\delta}^{*}}-P_{s}^{p, \tilde{\delta}^{*}}\right|^{2}\right\} .
$$

Since both terms on the right-hand side of (3.9) are treated similarly, we focus on the first one. Applying Itô's formula with $e^{\alpha s}\left|\hat{P}_{s}^{n, \tilde{\delta}^{*}}-P_{s}^{n, \tilde{\delta}^{*}}\right|^{2}$ and using the inequality $|x \vee y-x| \leq|x-y|, \forall x, y \in \mathbb{R}$, to deduce that: $\forall s \leq T$,

$$
\begin{aligned}
\mathbb{E}\left[e^{\alpha s}\left|\hat{P}_{s}^{n, \tilde{\delta}^{*}}-P_{s}^{n, \tilde{\delta}^{*}}\right|^{2}\right] & \\
\leq & \frac{2 C}{\alpha} \mathbb{E}\left[\int _ { s } ^ { T } e ^ { \alpha r } \left(\int_{E} \mid \gamma^{\tilde{\delta}^{*}}\left(X_{r}^{t, x}, e\right)\left\{u^{\tilde{\delta}^{*}, n-1}\left(r, X_{r^{-}}^{t, x}+\beta\left(X_{r^{-}}^{t, x}, e\right)\right)-u^{\tilde{\delta}^{*}, n-1}\left(r, X_{r^{-}}^{t, x}\right)\right.\right.\right. \\
& \left.\left.\left.\quad-u^{\tilde{\delta}^{*}, p-1}\left(r, X_{r^{-}}^{t, x}+\beta\left(X_{r^{-}}^{t, x}, e\right)\right)+u^{\tilde{\delta}^{*}, p-1}\left(r, X_{r^{-}}^{t, x}\right)\right\} \mid \lambda(d e)\right)^{2} d r\right],
\end{aligned}
$$

where $u^{\tilde{\delta}^{*}, n-1}(s,)=.u^{i, n-1}(s,$.$) if at time s, \tilde{\delta}_{s}^{*}=i$ and $C$ is a Lipschitz constant of the $\bar{f}_{i}^{\prime} s$ w.r.t $(\vec{y}, z, v)$ such that $\alpha \geq 2 C$. Next, using Cauchy-Schwarz inequality and (2.8) we get

$$
\begin{aligned}
& \mathbb{E}\left[e^{\alpha s} \mid \hat{P}_{s}^{n, \tilde{\delta}^{*}}-\right.\left.\left.P_{s}^{n, \tilde{\delta}^{*}}\right|^{2}\right] \\
& \leq \frac{2 C}{\alpha} \mathbb{E}\left[\int _ { s } ^ { T } e ^ { \alpha r } ( \int _ { E } | \gamma ^ { \tilde { \delta } ^ { * } } ( X _ { r } ^ { t , x } , e ) | ^ { 2 } \lambda ( d e ) ) \left(\int_{E} \mid u^{\tilde{\delta}^{*}, n-1}\left(r, X_{r^{-}}^{t, x}+\beta\left(X_{r^{-}}^{t, x}, e\right)\right)-u^{\tilde{\delta}^{*}, n-1}\left(r, X_{r^{-}}^{t, x}\right)\right.\right. \\
&\left.\left.\quad-u^{\tilde{\delta}^{*}, p-1}\left(r, X_{r^{-}}^{t, x}+\beta\left(X_{r^{-}}^{t, x}, e\right)\right)+\left.u^{\tilde{\delta}^{*}, p-1}\left(r, X_{r^{-}}^{t, x}\right)\right|^{2} \lambda(d e)\right) d r\right] \\
& \leq \frac{2 C}{\alpha} \mathbb{E}\left[\int _ { s } ^ { T } e ^ { \alpha r } \left(\int_{E} \mid u^{\tilde{\delta}^{*}, n-1}\left(r, X_{r^{-}}^{t, x}+\beta\left(X_{r^{-}}^{t, x}, e\right)\right)-u^{\tilde{\delta}^{*}, n-1}\left(r, X_{r^{-}}^{t, x}\right)\right.\right. \\
&\left.\left.\quad-u^{\tilde{\delta}^{*}, p-1}\left(r, X_{r^{-}}^{t, x}+\beta\left(X_{r^{-}}^{t, x}, e\right)\right)+\left.u^{\tilde{\delta}^{*}, p-1}\left(r, X_{r^{-}}^{t, x}\right)\right|^{2} \lambda(d e)\right) d r\right],
\end{aligned}
$$


for some constant $C$ (which may change from line to line) since $\lambda\left(\left(1 \wedge|e|^{2}\right)_{e \in E}\right)$ is finite. The exact same reasoning leads to the same estimate for $e^{\alpha s}\left|\hat{P}_{s}^{n, \tilde{\delta}^{*}}-P_{s}^{p, \tilde{\delta}^{*}}\right|^{2}$. Therefore, we deduce from (3.9) that:

$$
\begin{aligned}
\mathbb{E}\left[e^{\alpha s}\left|Y_{s}^{i, n}-Y_{s}^{i, p}\right|^{2}\right] & \\
\leq & \frac{4 C}{\alpha} \mathbb{E}\left[\int_{s}^{T} e^{\alpha r} \int_{E} \mid u^{i, n-1}\left(r, X_{r^{-}}^{t, x}+\beta\left(X_{r^{-}}^{t, x}, e\right)\right)-u^{i, n-1}\left(r, X_{r^{-}}^{t, x}\right)\right. \\
& \left.\left.\quad-u^{i, p-1}\left(r, X_{r^{-}}^{t, x}+\beta\left(X_{r^{-}}^{t, x}, e\right)\right)+u^{i, p-1}\left(r, X_{r^{-}}^{t, x}\right)\right\}\left.\right|^{2} \lambda(d e) d r\right] .
\end{aligned}
$$

Next, by taking $s=t$ in (3.10) and using the inequality $|a+b|^{2} \leq 2\left(|a|^{2}+|b|^{2}\right)$ for any real constants $a$ and $b$, we obtain:

$$
\begin{aligned}
& \left|u^{i, n}(t, x)-u^{i, p}(t, x)\right|^{2} \\
& \leq \frac{4 C}{\alpha} \mathbb{E}\left[\int_{t}^{T} e^{\alpha(r-t)} \int_{E} \mid u^{i, n-1}\left(r, X_{r^{-}}^{t, x}+\beta\left(X_{r^{-}}^{t, x}, e\right)\right)-u^{i, n-1}\left(r, X_{r^{-}}^{t, x}\right)\right. \\
& \left.\quad-u^{i, p-1}\left(r, X_{r^{-}}^{t, x}+\beta\left(X_{r^{-}}^{t, x}, e\right)\right)+\left.u^{i, p-1}\left(r, X_{r^{-}}^{t, x}\right)\right|^{2} \lambda(d e) d r\right] \\
& \leq \frac{8 C}{\alpha} \mathbb{E}\left[\int _ { t } ^ { T } e ^ { \alpha ( r - t ) } \int _ { E } \left\{\left|\left(u^{i, n-1}-u^{i, p-1}\right)\left(r, X_{r^{-}}^{t, x}+\beta\left(X_{r^{-}}^{t, x}, e\right)\right)\right|^{2}\right.\right. \\
& \left.\left.\quad+\left|\left(u^{i, n-1}-u^{i, p-1}\right)\left(r, X_{r^{-}}^{t, x}\right)\right|^{2}\right\} \lambda(d e) d r\right] .
\end{aligned}
$$

In order to take the supremum on the inequality (3.11), we need the boundedness of $\left(u^{i, n}\right)_{i \in \mathcal{I}}$. So we consider two cases. In the first one, we suppose that $f_{i}(t, x, 0,0,0)$ and $h_{i}(x)$ are bounded. Later on, we deal with the general case., i.e., without the boundedness of those latter functions.

Case 1: Assume that for any $i \in \mathcal{I}, h_{i}$ and $f_{i}(t, x, 0,0,0)$ are bounded. Then $u^{i, n}$ are uniformly bounded for any $i \in \mathcal{I}$ and $n \geq 0$. This can be obtained by the interpretation in terms of the value function of an optimal switching problem.

Now let us choose $\alpha=C$ and let $\eta$ be a constant such that $16 C^{-1}\left(e^{C \eta}-1\right)=\frac{1}{8}$. Note that $\eta$ does not depend on the terminal condition $\left(h_{i}\right)_{i \in \mathcal{I}}$. Finally let us set

$$
\left\|u^{i, n}-u^{i, p}\right\|_{\infty, \eta}:=\sup _{(t, x) \in[T-\eta, T] \times \mathbb{R}^{k}}\left|u^{i, n}(t, x)-u^{i, p}(t, x)\right| .
$$

Going back to $(3.11$ and taking the supremum over interval $[T-\eta, T]$, we deduce that for any $n, p \geq 1$,

$$
\begin{aligned}
\left\|u^{i, n}-u^{i, p}\right\|_{\infty, \eta}^{2} & \leq \underbrace{16 C^{-1}\left(e^{C \eta}-1\right)}_{=\frac{1}{8}} \sup _{(t, x) \in[T-\eta, T] \times \mathbb{R}^{k}}\left|u^{i, n}(t, x)-u^{i, p}(t, x)\right|^{2} \\
& =\frac{1}{8}\left\|u^{i, n-1}-u^{i, p-1}\right\|_{\infty, \eta}^{2}
\end{aligned}
$$

which means that the sequence $\left(\left(u^{i, n}\right)_{i \in \mathcal{I}}\right)_{n \geq 0}$ is uniformly convergent in $[T-\eta, T] \times \mathbb{R}^{k}$ such that for any $(t, x) \in[T-\eta, T] \times \mathbb{R}^{k}, u^{i}(t, x)=\lim _{n \rightarrow \infty} u^{i, n}(t, x)$. 
Next, let $t \in[T-2 \eta, T-\eta]$, then once more by (3.11), we have:

$$
\begin{aligned}
&\left|u^{i, n}(t, x)-u^{i, p}(t, x)\right|^{2} \\
& \leq \frac{8 C}{\alpha} \mathbb{E}\left[\int _ { t } ^ { T - \eta } e ^ { \alpha ( r - t ) } \int _ { E } \left\{\left|\left(u^{i, n-1}-u^{i, p-1}\right)\left(r, X_{r^{-}}^{t, x}+\beta\left(X_{r^{-}}^{t, x}, e\right)\right)\right|^{2}\right.\right. \\
&\left.\left.+\left|\left(u^{i, n-1}-u^{i, p-1}\right)\left(r, X_{r^{-}}^{t, x}\right)\right|^{2}\right\} \lambda(d e) d r\right] \\
&+\frac{8 C}{\alpha} \mathbb{E}\left[\int _ { T - \eta } ^ { T } e ^ { \alpha ( r - t ) } \int _ { E } \left\{\left|\left(u^{i, n-1}-u^{i, p-1}\right)\left(r, X_{r^{-}}^{t, x}+\beta\left(X_{r^{-}}^{t, x}, e\right)\right)\right|^{2}\right.\right. \\
&\left.\left.+\left|\left(u^{i, n-1}-u^{i, p-1}\right)\left(r, X_{r^{-}}^{t, x}\right)\right|^{2}\right\} \lambda(d e) d r\right]
\end{aligned}
$$

Then, if we choose $t=T-2 \eta$ and set

$$
\left\|u^{i, n}-u^{i, p}\right\|_{\infty, 2 \eta}:=\sup _{(t, x) \in[T-2 \eta, T-\eta] \times \mathbb{R}^{k}}\left|u^{i, n}(t, x)-u^{i, p}(t, x)\right|,
$$

we obtain:

$$
\begin{aligned}
\left\|u^{i, n}-u^{i, p}\right\|_{\infty, 2 \eta}^{2} & \leq 16 C^{-1}\left(\left(e^{C \eta}-1\right)\left\|u^{i, n-1}-u^{i, p-1}\right\|_{\infty, 2 \eta}^{2}+\left(e^{2 C \eta}-e^{C \eta}\right)\left\|u^{i, n-1}-u^{i, p-1}\right\|_{\infty, \eta}^{2}\right) \\
& \leq \frac{1}{8}\left\|u^{i, n-1}-u^{i, p-1}\right\|_{\infty, 2 \eta}^{2}+16 C^{-1}\left(e^{2 C \eta}-e^{C \eta}\right)\left\|u^{i, n-1}-u^{i, p-1}\right\|_{\infty, \eta}^{2} .
\end{aligned}
$$

It implies that

$$
\limsup _{n, p \rightarrow \infty}\left\|u^{i, n}-u^{i, p}\right\|_{\infty, 2 \eta}^{2} \leq \frac{1}{8} \limsup _{n, p \rightarrow \infty}\left\|u^{i, n-1}-u^{i, p-1}\right\|_{\infty, 2 \eta}^{2}
$$

since $\lim \sup _{n, p \rightarrow \infty}\left\|u^{i, n-1}-u^{i, p-1}\right\|_{\infty, \eta}^{2}=0$. Therefore

$$
\limsup _{n, p \rightarrow \infty}\left\|u^{i, n}-u^{i, p}\right\|_{\infty, 2 \eta}^{2}=0 .
$$

Thus, the sequence $\left(\left(u^{i, n}\right)_{i \in \mathcal{I}}\right)_{n \geq 0}$ is uniformly convergent in $[T-2 \eta, T-\eta] \times \mathbb{R}^{k}$. This implies the existence of deterministic continuous functions $\left(u^{i}\right)_{i \in \mathcal{I}}$ such that for any $i \in \mathcal{I}$ and $(t, x) \in[0, T] \times \mathbb{R}^{k}, u^{i, n}(t, x)$ converges w.r.t. $n$ to $u^{i}(t, x)$.

Continuing now this reasoning as many times as necessary on $[T-3 \eta, T-2 \eta],[T-4 \eta, T-3 \eta]$ etc. we obtain the uniform convergence of $\left(\left(u^{i, n}\right)_{i \in \mathcal{I}}\right)_{n \geq 0}$ in $[0, T] \times \mathbb{R}^{k}$.

Case 2: Here we deal with the general case. Firstly, by (H1)-iii), (H2) and (H3), there exist two constants $C$ and $p \in \mathbb{N}$ such $f_{i}(t, x, 0, \ldots, 0,0), h_{i}(x)$ and $g_{i j}(t, x)$ are of polynomial growth, i.e., for any $(t, x) \in[0, T] \times \mathbb{R}^{k}$,

$$
\left|f_{i}(t, x, 0, \ldots, 0,0)\right|+\left|h_{i}(x)\right|+\left|g_{i j}(t, x)\right| \leq C\left(1+|x|^{p}\right) .
$$

To proceed for $s \in[t, T]$ let us define,

$$
\bar{Y}_{s}^{i, n}:=Y_{s}^{i, n} \varphi\left(X_{s}^{t, x}\right)
$$

where for $x \in \mathbb{R}$,

$$
\varphi(x):=\frac{1}{\left(1+|x|^{2}\right)^{p}} .
$$


Then, by the integration-by-parts formula we have:

$$
\begin{aligned}
& d \bar{Y}_{s}^{i, n}=\varphi\left(X_{s}^{t, x}\right) d Y_{s}^{i, n}+Y_{s}^{i, n} d \varphi\left(X_{s}^{t, x}\right)+d\left\langle Y^{i, n}, \varphi\left(X^{t, x}\right)\right\rangle_{s} \\
& =\varphi\left(X_{s}^{t, x}\right)\left\{-f_{i}\left(s, X_{s}^{t, x},\left(Y_{s}^{k, n}\right)_{k \in \mathcal{I}}, Z_{s}^{i, n}, V_{s}^{i, n-1}\right) d s-d K_{s}^{i, n}+Z_{s}^{i, n} d B_{s}+\int_{E} V_{s}^{i, n}(e) \tilde{\mu}(d s, d e)\right\} \\
& +Y_{s}^{i, n}\left\{\left(\mathcal{L} \varphi\left(X_{s}^{t, x}\right)+K \varphi\left(X_{s}^{t, x}\right)\right) d s+D_{x} \varphi\left(X_{s}^{t, x}\right) \sigma\left(s, X_{s}^{t, x}\right) d B_{s}+\int_{E}\left(\varphi\left(X_{s^{-}}^{t, x}+\beta\left(X_{s^{-}}^{t, x}, e\right)\right)-\varphi\left(X_{s^{-}}^{t, x}\right)\right) \tilde{\mu}(d s, d e)\right\} \\
& +Z_{s}^{i, n} D_{x} \varphi\left(X_{s}^{t, x}\right) \sigma\left(s, X_{s}^{t, x}\right) d s+\int_{E} V_{s}^{i, n}(e)\left(\varphi\left(X_{s^{-}}^{t, x}+\beta\left(X_{s^{-}}^{t, x}, e\right)\right)-\varphi\left(X_{s^{-}}^{t, x}\right)\right) \lambda(d e) d s \\
& =\left\{-\varphi\left(X_{s}^{t, x}\right) f_{i}\left(s, X_{s}^{t, x},\left(Y_{s}^{k, n}\right)_{k \in \mathcal{I}}, Z_{s}^{i, n}, V_{s}^{i, n-1}\right)+\mathcal{L} \varphi\left(X_{s}^{t, x}\right) Y_{s}^{i, n}+K \varphi\left(X_{s}^{t, x}\right) Y_{s}^{i, n}+D_{x} \varphi\left(X_{s}^{t, x}\right) \sigma\left(s, X_{s}^{t, x}\right) Z_{s}^{i, n}\right. \\
& \left.+\int_{E} V_{s}^{i, n}(e)\left(\varphi\left(X_{s^{-}}^{t, x}+\beta\left(X_{s^{-}}^{t, x}, e\right)\right)-\varphi\left(X_{s^{-}}^{t, x}\right)\right) \lambda(d e)\right\} d s+\left\{Z_{s}^{i, n} \varphi\left(X_{s}^{t, x}\right)+Y_{s}^{i, n} D_{x} \varphi\left(X_{s}^{t, x}\right) \sigma\left(s, X_{s}^{t, x}\right)\right\} d B_{s} \\
& -\varphi\left(X_{s}^{t, x}\right) d K_{s}^{i, n}+\left\{\int_{E} V_{s}^{i, n}(e) \varphi\left(X_{s}^{t, x}\right)+Y_{s}^{i, n}\left(\varphi\left(X_{s^{-}}^{t, x}+\beta\left(X_{s^{-}}^{t, x}, e\right)\right)-\varphi\left(X_{s^{-}}^{t, x}\right)\right)\right\} \tilde{\mu}(d s, d e),
\end{aligned}
$$

where $\mathcal{L} \varphi$ and $K \varphi$ are given in (2.13)-(2.14). Next let us set, for $s \in[t, T]$,

$$
\begin{aligned}
& d \bar{K}_{s}^{i, n}:=\varphi\left(X_{s}^{t, x}\right) d K_{s}^{i, n} \\
& \bar{Z}_{s}^{i, n}:=Z_{s}^{i, n} \varphi\left(X_{s}^{t, x}\right)+Y_{s}^{i, n} D_{x} \varphi\left(X_{s}^{t, x}\right) \sigma\left(s, X_{s}^{t, x}\right), \quad \text { and } \\
& \bar{V}_{s}^{i, n}(e):=V_{s}^{i, n}(e) \varphi\left(X_{s}^{t, x}\right)+Y_{s}^{i, n}\left(\varphi\left(X_{s^{-}}^{t, x}+\beta\left(X_{s^{-}}^{t, x}, e\right)\right)-\varphi\left(X_{s^{-}}^{t, x}\right) .\right.
\end{aligned}
$$

Then $\left(\left(\bar{Y}^{i, n}, \bar{Z}^{i, n}, \bar{V}^{i, n}, \bar{K}^{i, n}\right)\right)_{i \in \mathcal{I}}$ verifies: $\forall s \in[t, T]$,

$$
\left\{\begin{array}{c}
\bar{Y}_{s}^{i, n}=\bar{h}_{i}\left(X_{T}^{t, x}\right)+\int_{s}^{T} \bar{f}_{i}\left(r, X_{r}^{t, x},\left(\bar{Y}_{r}^{k, n}\right)_{k \in \mathcal{I}}, \bar{Z}_{r}^{i, n}, \bar{V}_{r}^{i, n-1}\right) d r+\bar{K}_{T}^{i, n}-\bar{K}_{s}^{i, n} \\
\quad-\int_{s}^{T} \bar{Z}_{r}^{i, n} d B_{r}-\int_{s}^{T} \int_{E} \bar{V}_{r}^{i, n}(e) \tilde{\mu}(d r, d e), \\
\bar{Y}_{s}^{i, n} \geqslant \max _{j \in \mathcal{I}^{-i}}\left(\bar{Y}_{s}^{j, n}-\bar{g}_{i j}\left(s, X_{s}^{t, x}\right)\right) \\
\int_{0}^{T}\left(\bar{Y}_{s}^{i, n}-\max _{j \in \mathcal{I}^{-i}}\left(\bar{Y}_{s}^{j, n}-\bar{g}_{i j}\left(s, X_{s}^{t, x}\right)\right)\right) d \bar{K}_{s}^{i, n}=0,
\end{array}\right.
$$

where for any $i, j \in \mathcal{I}$,

$$
\bar{h}_{i}\left(X_{T}^{t, x}\right):=h_{i}\left(X_{T}^{t, x}\right) \varphi\left(X_{T}^{t, x}\right), \bar{g}_{i j}\left(s, X_{s}^{t, x}\right):=g_{i j}\left(s, X_{s}^{t, x}\right) \varphi\left(X_{s}^{t, x}\right),
$$

and

$$
\begin{aligned}
& \bar{f}_{i}(s, x, \vec{y}, z, v):= \\
& \varphi(x) f_{i}\left(s, x, \varphi^{-1}(x) \vec{y}, \varphi^{-1}(x)\left\{z-D_{x} \varphi(x) \sigma(s, x) \varphi^{-1}(x) y^{i}\right\}, \varphi^{-1}(x)\left\{v-\varphi^{-1}(x) y^{i}(\varphi(x+\beta(x, e))-\varphi(x))\right\}\right. \\
& \quad-\varphi^{-1}(x) y^{i} \mathcal{L} \varphi(x)-\varphi^{-1}(x) y^{i} K \varphi(x)-D_{x} \varphi(x) \sigma(s, x) \varphi^{-1}(x)\left\{z-D_{x} \varphi(x) \sigma(s, x) \varphi^{-1}(x) y^{i}\right\} \\
& \quad-\int_{E}(\varphi(x+\beta(x, e))-\varphi(x)) \varphi^{-1}(x)\left\{v-\varphi^{-1}(x) y^{i}(\varphi(x+\beta(x, e))-\varphi(x))\right\} \lambda(d e) .
\end{aligned}
$$

Here, let us notice that the functions $\bar{f}_{i}(t, x, 0,0,0), \bar{g}_{i j}$ and $\bar{h}_{i}$ are bounded and let us set

$$
\bar{u}^{i, n}(t, x):=\varphi(x) u^{i, n}(t, x) .
$$


Then by the result of the first case, the sequence $\left(\left(\bar{u}^{i, n}\right)_{i \in \mathcal{I}}\right)_{n>0}$ is uniformly convergent in $[0, T] \times \mathbb{R}^{k}$. Next it is enough to take $u^{i, n}(t, x):=\varphi^{-1}(x) \bar{u}^{i, n}(t, x)(t, x) \in[0, T] \times \mathbb{R}^{k}$ and $i \in \mathcal{I}$, which are uniformly convergent in compact sets of $[0, T] \times \mathbb{R}^{k}$.

We are now ready to study the convergence of the sequences $\left(Y^{i, n}, Z^{i, n}, V^{i, n}, K^{i, n}\right)_{n \geq 0}$. First, the sequence $\left(Y^{i, n}\right)_{n \geq 0}$ converges in $\mathcal{S}^{2}$ to $Y^{i}$. Actually, this can be obtained from the uniform convergence of $\left(u^{i, n}\right)_{n}$ to $u^{i}$ in compact sets of $[0, T] \times \mathbb{R}^{k}$, the definition (3.14) of $Y^{i, n}$ and the polynomial growth of $u^{i, n}$, that is

$$
\begin{aligned}
& \mathbb{E}\left[\sup _{s \leq T}\left|Y_{s}^{i, n}-Y_{s}^{i}\right|^{2}\right]=\mathbb{E}\left[\sup _{s \leq T}\left|\varphi^{-1}\left(X_{s}^{t, x}\right)\left(\bar{u}^{i, n}\left(s, X_{s}^{t, x}\right)-\bar{u}^{i}\left(s, X_{s}^{t, x}\right)\right)\right|^{2}\right] \\
& \leq \mathbb{E}\left[\sup _{s \leq T}\left\{\left|\varphi^{-1}\left(X_{s}^{t, x}\right)\left(\bar{u}^{i, n}\left(s, X_{s}^{t, x}\right)-\bar{u}^{i}\left(s, X_{s}^{t, x}\right)\right)\right|^{2} \mathbf{1}_{\left[\left|X_{s}^{t, x}\right|<h\right]}\right\}\right] \\
& +\mathbb{E}\left[\sup _{s \leq T}\left\{\left|\varphi^{-1}\left(X_{s}^{t, x}\right)\left(\bar{u}^{i, n}\left(s, X_{s}^{t, x}\right)-\bar{u}^{i}\left(s, X_{s}^{t, x}\right)\right)\right|^{2} \mathbf{1}_{\left[\left|X_{s}^{t, x}\right| \geq h\right]}\right\}\right] \\
& \leq C \sup _{(t, x) \in[0, T] \times B(0, h)}\left|\bar{u}^{i, n}(t, x)-\bar{u}^{i}(t, x)\right|^{2} \\
& +\mathbb{E}\left[\sup _{s \leq T}\left|\varphi^{-1}\left(X_{s}^{t, x}\right)\left(\bar{u}^{i, n}\left(s, X_{s}^{t, x}\right)-\bar{u}^{i}\left(s, X_{s}^{t, x}\right)\right)\right|^{2} \mathbf{1}_{\left[\sup _{s \leq T}\left|X_{s}^{t, x}\right| \geq h\right]}\right],
\end{aligned}
$$

where, for any $h>0, B(0, h)$ denotes the ball in $\mathbb{R}^{k}$ with center the origin and radius $h$. Obviously, the first term in the right-hand side of this inequality goes to 0 when $n \rightarrow \infty$. For the second term, using CauchySchwarz and Markov inequalities, we have

$$
\begin{aligned}
\mathbb{E}\left[\sup _{s \leq T} \mid \varphi^{-1}\left(X_{s}^{t, x}\right)\right. & \left.\left.\left(\bar{u}^{i, n}\left(s, X_{s}^{t, x}\right)-\bar{u}^{i}\left(s, X_{s}^{t, x}\right)\right)\right|^{2} \mathbf{1}_{\left[\sup _{s \leq T}\left|X_{s}^{t, x}\right| \geq h\right]}\right] \\
& \leq \mathbb{E}\left[\sup _{s \leq T}\left|\varphi^{-1}\left(X_{s}^{t, x}\right)\left(\bar{u}^{i, n}\left(s, X_{s}^{t, x}\right)-\bar{u}^{i}\left(s, X_{s}^{t, x}\right)\right)\right|^{2}\right]\left\{\mathbb{E}\left[\mathbf{1}_{\left[\sup _{s \leq T}\left|X_{s}^{t, x}\right|{ }^{2} \geq h\right]}\right]\right\}^{\frac{1}{2}} \\
& =\mathbb{E}\left[\sup _{s \leq T}\left|\varphi^{-1}\left(X_{s}^{t, x}\right)\left(\bar{u}^{i, n}\left(s, X_{s}^{t, x}\right)-\bar{u}^{i}\left(s, X_{s}^{t, x}\right)\right)\right|^{2}\right]\left\{\mathbb{P}\left[\sup _{s \leq T}\left|X_{s}^{t, x}\right|^{2} \geq h\right]\right\}^{\frac{1}{2}} \\
& \leq E\left[\sup _{s \leq T}\left|\varphi^{-1}\left(X_{s}^{t, x}\right)\left(\bar{u}^{i, n}\left(s, X_{s}^{t, x}\right)-\bar{u}^{i}\left(s, X_{s}^{t, x}\right)\right)\right|^{2}\right]\left\{\frac{\mathbb{E}\left[\sup _{s \leq T}\left|X_{s}^{t, x}\right|^{2}\right]}{h}\right\}^{\frac{1}{2}} .
\end{aligned}
$$

Next, for any fixed $\epsilon>0$, there exists $h_{\epsilon} \leq \epsilon$ such that $\left\{\frac{\mathbb{E}\left[\sup _{s \leq T}\left|X_{s}^{t, x}\right|^{2}\right]}{h}\right\}^{\frac{1}{2}} \leq \epsilon$. Finally, taking the limit superior as $n \rightarrow \infty$ in 3.17 to obtain

$$
\limsup _{n \rightarrow \infty} \mathbb{E}\left[\sup _{s \leq T}\left|Y_{s}^{i, n}-Y_{s}^{i}\right|^{2}\right] \leq \epsilon .
$$

As $\epsilon$ is arbitrary, then

$$
\limsup _{n \rightarrow \infty} \mathbb{E}\left[\sup _{s \leq T}\left|Y_{s}^{i, n}-Y_{s}^{i}\right|^{2}\right]=0 .
$$

On the other hand, as the measure $\lambda$ is finite and by the characterization 3.3 of the sequence $\left(V^{i, n}\right)_{n \geq 0}$ by means of the function $\left(u^{i, n}\right)_{n \geq 0}$ and the uniform convergence of $\left(u^{i, n}\right)_{n \geq 0}$, the sequence $\left(V^{i, n}\right)_{n \geq 0}$ converges in $\mathcal{H}^{2}\left(\mathcal{L}^{2}(\lambda)\right)$ to $V^{i}$.

Next, we focus on the convergence of the components $\left(Z^{i, n}, K^{i, n}\right)_{n \geq 0}$. For this, we first establish a priori 
estimates, uniform on $n$ on the sequences $\left(Z^{i, n}, K^{i, n}\right)_{n \geq 0}$. Applying Itô's formula to $\left|Y_{s}^{i, n}\right|^{2}$, we have:

$$
\begin{aligned}
& \mathbb{E}\left[\left|Y_{s}^{i, n}\right|^{2}\right]+ \mathbb{E}\left[\int_{s}^{T}\left|Z_{r}^{i, n}\right|^{2} d r\right]+\mathbb{E}\left[\int_{s}^{T} \int_{E}\left|V_{r}^{i, n}(e)\right|^{2} \lambda(d e) d r\right] \\
&=\mathbb{E}\left[\mid h_{i}\left(\left.X_{T}^{t, x}\right|^{2}\right]+2 \mathbb{E}\left[\int_{s}^{T} Y_{r}^{i, n} f_{i}\left(r, X_{r}^{t, x},\left(Y_{r}^{k, n}\right)_{k \in \mathcal{I}}, Z_{r}^{i, n}, V_{r}^{i, n-1}\right) d r\right]\right. \\
&+2 \mathbb{E}\left[\int_{s}^{T} Y_{r}^{i, n} d K_{r}^{i, n}\right] .
\end{aligned}
$$

Then by a linearization procedure of $f_{i}$, which is possible since it is Lipschitz w.r.t $(\vec{y}, z, q)$ and using the inequality $2 a b \leq \frac{1}{\epsilon} a^{2}+\epsilon b^{2}$ for any constant $\epsilon>0$, we have:

$$
\begin{aligned}
\mathbb{E}\left[\int_{0}^{T}\left|Z_{r}^{i, n}\right|^{2} d r\right] \leq \mathbb{E}\left[\mid h_{i}\left(\left.X_{T}^{t, x}\right|^{2}\right]+2 \mathbb{E}\left[\int _ { 0 } ^ { T } | Y _ { r } ^ { i , n } | \left\{\left|f_{i}\left(r, X_{r}^{t, x}, 0,0,0\right)\right|\right.\right.\right. \\
\left.\left.+\sum_{l=1, m} a_{r}^{i, l}\left|Y_{r}^{l}\right|+b_{r}^{i, l}\left|Z_{r}^{i}\right|+\int_{E} c_{r}^{i, l}(e)\left|V_{r}^{i, n-1}(e)\right| \lambda(d e)\right\} d r\right] \\
+\frac{1}{\epsilon} \mathbb{E}\left[\sup _{s \leq T}\left|Y_{s}^{i, n}\right|^{2}\right]+\epsilon \mathbb{E}\left[\left(K_{T}^{i, n}\right)^{2}\right],
\end{aligned}
$$

where $a^{i, l} \in \mathbb{R}, b^{i, l} \in \mathbb{R}^{d}$ are $\mathcal{P}$-measurable processes and $c^{i, l} \in \mathbb{R}$ is $\mathcal{P} \otimes \mathcal{E}$-measurable process, bounded by the Lipschitz constant of $f_{i}$. Using again the inequality $2 a b \leq \frac{1}{\nu} a^{2}+\nu b^{2}$ for $\nu>0$ yields

$$
\begin{aligned}
\mathbb{E}\left[\int_{0}^{T}\left|Z_{r}^{i, n}\right|^{2} d r\right] \leq \mathbb{E}[ & \mid h_{i}\left(\left.X_{T}^{t, x}\right|^{2}\right]+\frac{1}{\nu} \mathbb{E}\left[\int_{0}^{T}\left|Y_{r}^{i, n}\right|^{2} d r\right]+\nu \mathbb{E}\left[\int _ { 0 } ^ { T } \left\{\left|f_{i}\left(r, X_{r}^{t, x}, 0,0,0\right)\right|\right.\right. \\
& \left.\left.+\sum_{l=1, m} a_{r}^{i, l}\left|Y_{r}^{l, n}\right|+b_{r}^{i, l}\left|Z_{r}^{i, n}\right|+\int_{E} c_{r}^{i, l}(e)\left|V_{r}^{i, n-1}(e)\right| \lambda(d e)\right\}^{2} d r\right] \\
& +\frac{1}{\epsilon} \mathbb{E}\left[\sup _{s \leq T}\left|Y_{s}^{i, n}\right|^{2}\right]+\epsilon \mathbb{E}\left[\left(K_{T}^{i, n}\right)^{2}\right] .
\end{aligned}
$$

From the polynomial growth condition on $f_{i}$ and $h_{i}$, and since $|a+b+c+d|^{2} \leq 4\left\{|a|^{2}+|b|^{2}+|c|^{2}+|d|^{2}\right\}$ for any real constants $a, b, c$ and $d$, we have :

$$
\begin{aligned}
\mathbb{E}\left[\int_{0}^{T}\left|Z_{r}^{i, n}\right|^{2} d r\right] \leq & C_{1}\left(1+\mathbb{E}\left[\left|X_{T}^{t, x}\right|^{2 p}\right]\right)+\frac{1}{\nu} \mathbb{E}\left[\int_{0}^{T}\left|Y_{r}^{i, n}\right|^{2} d r\right] \\
& +4 \nu C_{2}\left(1+\mathbb{E}\left[\int_{0}^{T}\left|X_{r}^{t, x}\right|^{2 p} d r\right]\right)+4 \nu C_{3} \mathbb{E}\left[\int_{0}^{T} \sum_{l=1, m}\left|Y_{r}^{l, n}\right|^{2} d r\right] \\
& +4 \nu C_{3} \mathbb{E}\left[\int_{0}^{T}\left|Z_{r}^{i, n}\right|^{2} d r\right]+4 \nu C_{3} \mathbb{E}\left[\int_{0}^{T} \int_{E}\left|V_{r}^{i, n-1}(e)\right|^{2} \lambda(d e) d r\right] \\
& +\frac{1}{\epsilon} \mathbb{E}\left[\sup _{s \leq T}\left|Y_{s}^{i, n}\right|^{2}\right]+\epsilon \mathbb{E}\left[\left(K_{T}^{i, n}\right)^{2}\right],
\end{aligned}
$$


for suitable positive constants $C_{1}, C_{2}$ and $C_{3}$. Now, by estimate (2.5) (with $4 \nu C_{3}<1$ ), and taking the summation over all $i \in \mathcal{I}$, we obtain

$$
\begin{aligned}
& \sum_{i=1, m} \mathbb{E}\left[\int_{0}^{T}\left|Z_{r}^{i, n}\right|^{2} d r\right] \leq C\left(1+|x|^{2 p}+\sum_{i=1, m} \mathbb{E}\left[\sup _{s \leq T}\left|Y_{s}^{i, n}\right|^{2}\right]+\sum_{i=1, m} \mathbb{E}\left[\int_{0}^{T} \int_{E}\left|V_{r}^{i, n-1}(e)\right|^{2} \lambda(d e) d r\right]\right) \\
&+\epsilon \sum_{i=1, m} \mathbb{E}\left[\left(K_{T}^{i, n}\right)^{2}\right]
\end{aligned}
$$

where $C=C(T, m, \nu, \epsilon)>0$ is a constant independent of $n$, which may change from line to line.

Through the convergence of $\left(Y^{i, n}\right)_{n}$ in $\mathcal{S}^{2}$, we have $\sup _{n \geq 0} \mathbb{E}\left[\sup _{s \leq T}\left|Y_{s}^{i, n}\right|^{2}\right] \leq C$, and then taking into consideration the convergence of $\left(V^{i, n}\right)_{n}$ in $\mathcal{H}^{2}\left(\mathcal{L}^{2}(\lambda)\right)$, we finally obtain

$$
\sum_{i=1, m} \mathbb{E}\left[\int_{0}^{T}\left|Z_{r}^{i, n}\right|^{2} d r\right] \leq C\left(1+|x|^{2 p}\right)+\epsilon \sum_{i=1, m} \mathbb{E}\left[\left(K_{T}^{i, n}\right)^{2}\right] .
$$

Now, from the relation

$$
\begin{aligned}
K_{T}^{i, n}=Y_{0}^{i, n} & -h_{i}\left(X_{T}^{t, x}\right)-\int_{0}^{T} f_{i}\left(r, X_{r}^{t, x},\left(Y_{r}^{k, n}\right)_{\left.k \in \mathcal{I}, Z_{r}^{i, n}, V_{r}^{i, n-1}\right) d r}\right. \\
& +\int_{0}^{T} Z_{r}^{i, n} d B_{r}+\int_{0}^{T} \int_{E} V_{r}^{i, n}(e) \tilde{\mu}(d r, d e),
\end{aligned}
$$

and, once again, by a linearization procedure of Lipschitz function $f_{i}$ and the polynomial growth condition on $f_{i}(t, x, 0,0,0)$ and $h_{i}(x)$, there exist some positive constant $C^{\prime}$ such that

$$
\begin{gathered}
\sum_{i=1, m} \mathbb{E}\left[\left(K_{T}^{i, n}\right)^{2}\right] \leq C^{\prime}\left(1+|x|^{2 p}+\sum_{i=1, m} \mathbb{E}\left[\sup _{s \leq T}\left|Y_{s}^{i, n}\right|^{2}\right]+\sum_{i=1, m} \mathbb{E}\left[\int_{0}^{T}\left|Z_{r}^{i, n}\right|^{2} d r\right]\right. \\
\left.+\sum_{i=1, m} \mathbb{E}\left[\int_{0}^{T} \int_{E}\left|V_{r}^{i, n-1}(e)\right|^{2} \lambda(d e) d r\right]\right) \\
\leq C^{\prime}\left(1+|x|^{2 p}+\sum_{i=1, m} \mathbb{E}\left[\int_{0}^{T}\left|Z_{r}^{i, n}\right|^{2} d r\right]\right) .
\end{gathered}
$$

Combining this last estimate with (3.18) and choosing $\epsilon$ small enough since it is arbitrary, then we obtain a constant $\bar{C}_{t x}$ which may depend on $t$ and $x$ such that

$$
\sum_{i=1, m} \mathbb{E}\left[\int_{0}^{T}\left|Z_{r}^{i, n}\right|^{2} d r+\left(K_{T}^{i, n}\right)^{2}\right] \leq \bar{C}_{t x} .
$$

Now, for any $n, p \geq 1$, it follows from Itô's formula that

$$
\begin{aligned}
& \mathbb{E}\left[\int_{0}^{T}\left|Z_{r}^{i, n}-Z_{r}^{i, p}\right|^{2} d r\right] \leq 2 \mathbb{E}\left[\int _ { 0 } ^ { T } ( Y _ { r } ^ { i , n } - Y _ { r } ^ { i , p } ) \left(f_{i}\left(r, X_{r}^{t, x},\left(Y_{r}^{k, n}\right)_{k \in \mathcal{I}}, Z_{r}^{i, n}, V_{r}^{i, n-1}\right)\right.\right. \\
&-f_{i}\left(r, X_{r}^{t, x},\left(Y_{r}^{k, p}\right)_{\left.\left.\left.k \in \mathcal{I}, Z_{r}^{i, p}, V_{r}^{i, p-1}\right)\right) d r\right]}\right. \\
&+ 2 \mathbb{E}\left[\int_{0}^{T}\left(Y_{r}^{i, n}-Y_{r}^{i, p}\right)\left(d K_{r}^{i, n}(r)-d K_{r}^{i, p}\right)\right] .
\end{aligned}
$$


By Cauchy-Schwarz inequality and using the inequality $2 a b \leq \frac{1}{\eta} a^{2}+\eta b^{2}$ for $\eta>0$, we have

$$
\begin{aligned}
& \mathbb{E}\left[\int_{0}^{T}\left|Z_{r}^{i, n}-Z_{r}^{i, p}\right|^{2} d r\right] \leq 2 \sqrt{\mathbb{E}\left[\sup _{s \leq T}\left|Y_{r}^{i, n}-Y_{r}^{i, p}\right|^{2}\right]} \times \\
& \quad \sqrt{\mathbb{E}\left[\int_{0}^{T}\left|f_{i}\left(r, X_{r}^{t, x},\left(Y_{r}^{k, n}\right)_{k \in \mathcal{I}}, Z_{r}^{i, n}, V_{r}^{i, n-1}\right)-f_{i}\left(r, X_{r}^{t, x},\left(Y_{r}^{k, p}\right)_{k \in \mathcal{I}}, Z_{r}^{i, p}, V_{r}^{i, p-1}\right)\right|^{2} d r\right]} \\
& \quad+\frac{1}{\eta} \mathbb{E}\left[\sup _{s \leq T}\left|Y_{s}^{i, n}-Y_{s}^{i, p}\right|^{2}\right]+\eta \mathbb{E}\left[\left(K_{T}^{i, n}+K_{T}^{i, p}\right)^{2}\right] .
\end{aligned}
$$

But there exists a constant $C \geq 0$ (independent of $n$ and $p$ ) such that, for all $n, p \geq 1$,

$$
\mathbb{E}\left[\int_{0}^{T}\left|f_{i}\left(r, X_{r}^{t, x},\left(Y_{r}^{k, n}\right)_{k \in \mathcal{I}}, Z_{r}^{i, n}, V_{r}^{i, n-1}\right)-f_{i}\left(r, X_{r}^{t, x},\left(Y_{r}^{k, p}\right)_{k \in \mathcal{I}}, Z_{r}^{i, p}, V_{r}^{i, p-1}\right)\right|^{2} d r\right] \leq C .
$$

From the converges result of $Y^{i, n}$ in $\mathcal{S}^{2}, 3.20$ and (3.21), we deduce that:

$$
\mathbb{E}\left[\int_{0}^{T}\left|Z_{r}^{i, n}-Z_{r}^{i, p}\right|^{2} d r\right] \rightarrow 0 \text { as } n, p \rightarrow \infty
$$

This implies that $\left(Z^{i, n}\right)_{n \geq 0}$ is a Cauchy sequence in complete space, then there exists a process $Z^{i}, \mathcal{F}_{t^{-}}$ progressively measurable such that the sequence $\left(Z^{i, n}\right)_{n \geq 0}$ converges in $\mathcal{H}^{2, d}$ to $Z^{i}$. Finally, since for $s \leq T$,

$$
\begin{aligned}
K_{s}^{i, n}=Y_{0}^{i, n} & -Y_{s}^{i, n}-\int_{0}^{s} f_{i}\left(r, X_{r}^{t, x},\left(Y_{r}^{k, n}\right)_{k \in \mathcal{I}}, Z_{r}^{i, n}, V_{r}^{i, n-1}\right) d r \\
& +\int_{0}^{s} Z_{r}^{i, n} d B_{r}+\int_{0}^{s} \int_{E} V_{r}^{i, n}(e) \tilde{\mu}(d r, d e),
\end{aligned}
$$

then we have also $\mathbb{E}\left[\sup _{s \leq T}\left|K_{s}^{i, n}-K_{s}^{i, p}\right|^{2} d r\right] \rightarrow 0$ as $n, p \rightarrow \infty$. Thus, there exist $\mathcal{F}_{t}$-adapted nondecreasing and continuous process $\left(K_{s}\right)_{s \leq T}$ such that $\mathbb{E}\left[\sup _{s \leq T}\left|K_{s}^{i, n}-K_{s}^{i}\right|^{2} d r\right] \rightarrow 0$ as $n \rightarrow \infty$.

Finally, let us show that the third condition in (3.1) is satisfied by $\left(Y^{i}, Z^{i}, V^{i}, K^{i}\right)_{i \in \mathcal{I}}$. Now

$$
\begin{gathered}
\int_{0}^{T}\left(Y_{s}^{i}-\max _{j \in \mathcal{I}^{-i}}\left(Y_{s}^{j}-g_{i j}\left(s, X_{s}^{t, x}\right)\right)\right) d K_{s}^{i}=\int_{0}^{T}\left(Y_{s}^{i}-\max _{j \in \mathcal{I}^{-i}}\left(Y_{s}^{j}-g_{i j}\left(s, X_{s}^{t, x}\right)\right)\right)\left(d K_{s}^{i}-d K_{s}^{i, n}\right) \\
+\int_{0}^{T}\left(Y_{s}^{i}-\max _{j \in \mathcal{I}^{-i}}\left(Y_{s}^{j}-g_{i j}\left(s, X_{s}^{t, x}\right)\right)\right) d K_{s}^{i, n} .
\end{gathered}
$$

Let $\omega$ be fixed. It follows from the uniform convergence of $\left(Y^{i, n}\right)_{n}$ to $\left(Y^{i}\right)_{i \in \mathcal{I}}$ that, for any $\epsilon \geq 0$, there exist $N_{\epsilon}(\omega) \in \mathbb{N}$, such that for any $n \geq N_{\epsilon}(\omega)$ and $s \leq T$,

$$
Y_{s}^{i}(\omega)-\max _{j \in \mathcal{I}^{-i}}\left(Y_{s}^{j}(\omega)-g_{i j}\left(\omega, s, X_{s}^{t, x}\right)\right) \leq Y_{s}^{i, n}(\omega)-\max _{j \in \mathcal{I}^{-i}}\left(Y_{s}^{j, n}(\omega)-g_{i j}\left(\omega, s, X_{s}^{t, x}\right)\right)+\epsilon .
$$

Therefore, for $n \geq N_{\epsilon}(\omega)$ we have

$$
\int_{0}^{T}\left(Y_{s}^{i}-\max _{j \in \mathcal{I}^{-i}}\left(Y_{s}^{j}-g_{i j}\left(s, X_{s}^{t, x}\right)\right)\right) d K_{s}^{i, n} \leq \epsilon K_{T}^{i}(\omega) .
$$


On the other hand, since the function

$$
Y^{i}(\omega)-\max _{j \in \mathcal{I}^{-i}}\left(Y^{j}(\omega)-g_{i j}(\omega, ., .)\right):(t, x) \in[0, T] \times \mathbb{R}^{k} \longmapsto Y_{t}^{i}(\omega)-\max _{j \in \mathcal{I}^{-i}}\left(Y_{t}^{j}(\omega)-g_{i j}(\omega, t, x)\right)
$$

is $r c l l$ and then bounded. Then, there exists a sequence of step functions $\left(f^{m}(\omega, ., .)\right)_{m}$ which converges uniformly on $[0, T] \times \mathbb{R}^{k}$ to $Y^{i}(\omega)-\max _{j \in \mathcal{I}^{-i}}\left(Y^{j}(\omega)-g_{i j}(\omega, . .).\right)$, i.e., there exist $m_{\epsilon}(\omega) \geq 0$ such that for $m \geq m_{\epsilon}(\omega)$, we have

$$
\left|Y_{t}^{i}(\omega)-\max _{j \in \mathcal{I}^{-i}}\left(Y_{t}^{j}(\omega)-g_{i j}(\omega, t, x)\right)-f^{m}(\omega, t, x)\right|<\epsilon
$$

It follows that

$$
\begin{aligned}
& \int_{0}^{T}\left(Y_{s}^{i}-\max _{j \in \mathcal{I}^{-i}}\left(Y_{s}^{j}-g_{i j}\left(s, X_{s}^{t, x}\right)\right)\right)\left(d K_{s}^{i}-d K_{s}^{i, n}\right) \\
& =\int_{0}^{T}\left(Y_{s}^{i}-\max _{j \in \mathcal{I}^{-i}}\left(Y_{s}^{j}-g_{i j}\left(s, X_{s}^{t, x}\right)\right)-f^{m}\left(\omega, s, X_{s}^{t, x}\right)\right)\left(d K_{s}^{i}-d K_{s}^{i, n}\right)+\int_{0}^{T} f^{m}\left(\omega, s, X_{s}^{t, x}\right)\left(d K_{s}^{i}-d K_{s}^{i, n}\right) \\
& \leq \int_{0}^{T} f^{m}\left(\omega, s, X_{s}^{t, x}\right)\left(d K_{s}^{i}-d K_{s}^{i, n}\right)+\epsilon\left(K_{T}^{i}(\omega)+K_{T}^{i, n}(\omega)\right) .
\end{aligned}
$$

But the right-hand side converges to $2 \epsilon K_{T}^{i}(\omega)$, as $n \rightarrow \infty$, since $f^{m}(\omega, .$.$) is a step function and then$ $\int_{0}^{T} f^{m}\left(\omega, s, X_{s}^{t, x}\right)\left(d K_{s}^{i}-d K_{s}^{i, n}\right) \rightarrow 0$. Therefore, we have

$$
\limsup _{n \rightarrow \infty} \int_{0}^{T}\left(Y_{s}^{i}-\max _{j \in \mathcal{I}^{-i}}\left(Y_{s}^{j}-g_{i j}\left(s, X_{s}^{t, x}\right)\right)\right)\left(d K_{s}^{i}-d K_{s}^{i, n}\right) \leq 2 \epsilon K_{T}^{i}(\omega) .
$$

Finally, from (3.22), 3.23 and 3.24 we deduce that

$$
\int_{0}^{T}\left(Y_{s}^{i}-\max _{j \in \mathcal{I}^{-i}}\left(Y_{s}^{j}-g_{i j}\left(s, X_{s}^{t, x}\right)\right)\right) d K_{s}^{i} \leq 3 \epsilon K_{T}^{i}(\omega) .
$$

As $\epsilon$ is arbitrary and $Y_{s}^{i} \geq \max _{j \in \mathcal{I}^{-i}}\left(Y_{s}^{j}-g_{i j}\left(s, X_{s}^{t, x}\right)\right)$, then

$$
\int_{0}^{T}\left(Y_{s}^{i}-\max _{j \in \mathcal{I}^{-i}}\left(Y_{s}^{j}-g_{i j}\left(s, X_{s}^{t, x}\right)\right)\right) d K_{s}^{i}=0
$$

which completes the proof.

As a by-product of the Theorem 3.2 we have the following

Corollary 3.3 There exist deterministic continuous functions $\left(u^{i}\right)_{i \in \mathcal{I}}$ of polynomial growth, defined on $[0, T] \times$ $\mathbb{R}^{k}$, such that:

$$
\forall s \in[t, T], Y_{s}^{i, t, x}=u^{i}\left(s, X_{s}^{t, x}\right),
$$

and

$$
V_{s}^{i, t, x}(e)=u^{i}\left(s, X_{s^{-}}^{t, x}+\beta\left(X_{s^{-}}^{t, x}, e\right)\right)-u^{i}\left(s, X_{s^{-}}^{t, x}\right), d s \otimes d \mathbb{P} \otimes d \lambda \text { on }[t, T] \times \Omega \times E .
$$

Now, we provide the uniqueness of the markovian solution to reflected BSDEs 3.1. 
Proposition 3.4 Let $\left(\tilde{u}^{i}\right)_{i \in \mathcal{I}}$ be the deterministic continuous functions of polynomial growth such that

$$
\forall s \in[t, T], Y_{s}^{i, t, x}=\tilde{u}^{i}\left(s, X_{s}^{t, x}\right) .
$$

Then, for any $i \in \mathcal{I}, \tilde{u}^{i}=u^{i}$.

Proof: In order to show that the markovian solution to reflected BSDEs is unique [3.1), we suppose that there exists another continuous with polynomial growth functions $\left(\tilde{u}^{i}\right)_{i \in \mathcal{I}}$ such that:

$$
\forall s \in[t, T], \tilde{Y}_{s}^{i, t, x}=\tilde{u}^{i}\left(s, X_{s}^{t, x}\right),
$$

where $\left(\tilde{Y}^{i, t, x}\right)_{i \in \mathcal{I}}$ is the first component of the solution of the following system of RBSDEs with jumps with interconnected obstacles (3.1): for any $i \in \mathcal{I}$,

$$
\left\{\begin{aligned}
& \tilde{Y}^{i, t, x} \in \mathcal{S}^{2}, \tilde{Z}^{i, t, x} \in \mathcal{H}^{2, d}, \tilde{V}^{i, t, x} \in \mathcal{H}^{2}\left(\mathcal{L}^{2}(\lambda)\right), \text { and } \tilde{K}^{i, t, x} \in \mathcal{A}^{2} ; \\
& \tilde{Y}_{s}^{i, t, x}= h_{i}\left(X_{T}^{t, x}\right)+\int_{s}^{T} \bar{f}_{i}\left(r, X_{r}^{t, x},\left(\tilde{Y}_{r}^{k, t, x}\right)_{k \in \mathcal{I}}, \tilde{Z}_{r}^{i, t, x}, \int_{E} \gamma_{i}\left(X_{r}^{t, x}, e\right) \tilde{V}_{r}^{i, t, x}(e) \lambda(d e)\right) d r \\
& \quad+\tilde{K}_{T}^{i, t, x}-\tilde{K}_{s}^{i, t, x}-\int_{s}^{T} \tilde{Z}_{r}^{i, t, x} d B_{r}-\int_{s}^{T} \int_{E} \tilde{V}_{r}^{i, t, x}(e) \tilde{\mu}(d r, d e), \\
& \tilde{Y}_{s}^{i, t, x} \geqslant \max _{j \in \mathcal{I}^{-i}}\left(\tilde{Y}_{s}^{j, t, x}-g_{i j}\left(s, X_{s}^{t, x}\right)\right) \text { and } \int_{0}^{T}\left(\tilde{Y}_{s}^{i, t, x}-\max _{j \in \mathcal{I}^{-i}}\left(\tilde{Y}_{s}^{j, t, x}-g_{i j}\left(s, X_{s}^{t, x}\right)\right)\right) d \tilde{K}_{s}^{i, t, x}=0 .
\end{aligned}\right.
$$

On the other hand, as for any $i \in\{1, \ldots m\}, \tilde{u}^{i}$ is continuous function of polynomial growth and due to the finiteness of $\lambda$, one has

$$
\tilde{V}_{s}^{i, t, x}(e)=\tilde{u}^{i}\left(s, X_{s^{-}}^{t, x}+\beta\left(X_{s^{-}}^{t, x}, e\right)\right)-\tilde{u}^{i}\left(s, X_{s^{-}}^{t, x}\right), d s \otimes d \mathbb{P} \otimes d \lambda \text { on }[t, T] \times \Omega \times E .
$$

Now, let us consider the triplet of processes $\left(P^{\delta}, N^{\delta}, Q^{\delta}\right)$ associated with admissible strategy $\delta \in \mathcal{A}_{s}^{i}$ and which solves the following BSDE:

$$
P_{s}^{\delta}=h^{\delta}\left(X_{T}^{t, x}\right)+\int_{s}^{T} f^{\delta}\left(r, X_{r}^{t, x}\right) d r-\int_{s}^{T} N_{r}^{\delta} d B_{r}-\int_{s}^{T} \int_{E} Q_{r}^{\delta}(e) \tilde{\mu}(d r, d e)-A_{T}^{\delta}+A_{s}^{\delta}, s \leq T,
$$

where, for $\delta_{s}=i, f^{\delta}\left(s, X_{s}^{t, x}\right)$ is equal to

$$
\bar{f}_{i}\left(s, X_{s}^{t, x},\left(\tilde{u}^{k}\left(s, X_{s}^{t, x}\right)_{k \in \mathcal{I}}, \tilde{Z}_{s}^{i}, \int_{E} \gamma_{i}\left(X_{s}^{t, x}, e\right)\left\{\tilde{u}^{i}\left(s, X_{s^{-}}^{t, x}+\beta\left(X_{s^{-}}^{t, x}, e\right)\right)-\tilde{u}^{i}\left(s, X_{s^{-}}^{t, x}\right)\right\} \lambda(d e)\right) .\right.
$$

Therefore, we have the following representation of $\tilde{Y}^{i}$ :

$$
\tilde{Y}_{s}^{i}=\operatorname{esssup}_{\delta \in \mathcal{A}_{s}^{i}}\left(P_{s}^{\delta}-A_{s}^{\delta}\right), \quad s \leq T .
$$

Next, by using the inequality (3.11, we deduce that for any $i \in \mathcal{I}$,

$$
\begin{gathered}
\left|u^{i}(t, x)-\tilde{u}^{i}(t, x)\right|^{2} \leq \frac{8 C}{\alpha} \mathbb{E}\left[\int _ { t } ^ { T } e ^ { \alpha ( r - t ) } \int _ { E } \left\{\left|\left(u^{i}-\tilde{u}^{i}\right)\left(r, X_{r^{-}}^{t, x}+\beta\left(X_{r^{-}}^{t, x}, e\right)\right)\right|^{2}\right.\right. \\
\left.\left.+\mid\left(u^{i}-\tilde{u}^{i}\right)\left(r, X_{r^{-}}^{t, x}\right)\right\}\left.\right|^{2} \lambda(d e) d r\right] .
\end{gathered}
$$

Here, we follow the same approach as in the proof of Theorem (3.2), i.e., we consider two cases. In the first one, we assume that $\tilde{f}_{i}(t, x, 0,0,0)$ and $h_{i}(x)$ are bounded, then the deterministic functions $u^{i}$ and $\tilde{u}^{i}$ are also bounded. Latter on we deal with the general case. 
$\underline{\text { Case } 1}$ : Recall that $\eta$ does not depend on the terminal condition $\left(h_{i}\right)_{i \in \mathcal{I}}$ and $16 C^{-1}\left(e^{C \eta}-1\right)=\frac{1}{8}$. Then, we deduce from (3.11), that for any $i \in \mathcal{I}$,

$$
\left\|u^{i}-\tilde{u}^{i}\right\|_{\infty, \eta}^{2} \leq \frac{1}{8}\left\|u^{i}-\tilde{u}^{i}\right\|_{\infty, \eta}^{2}
$$

which implies that, for any $i \in \mathcal{I}, u^{i}=\tilde{u}^{i}$ on $[T-\eta, T]$. Consequently, for any $s \in[T-\eta, T]$ and $i \in \mathcal{I}$, $Y_{s}^{i, t, x}=\tilde{Y}_{s}^{i, t, x}$.

Next, on $[T-2 \eta, T-\eta]$, we have

$$
\left\|u^{i}-\tilde{u}^{i}\right\|_{\infty, 2 \eta}^{2} \leq \frac{1}{8}\left\|u^{i}-\tilde{u}^{i}\right\|_{\infty, 2 \eta}^{2}+16 C^{-1}\left(e^{2 C \eta}-e^{C \eta}\right)\left\|u^{i}-\tilde{u}^{i}\right\|_{\infty, \eta}^{2} .
$$

Since $u^{i}=\tilde{u}^{i}$ on $[T-\eta, T]$, we then obtain:

$$
\left\|u^{i}-\tilde{u}^{i}\right\|_{\infty, 2 \eta}^{2} \leq \frac{1}{8}\left\|u^{i}-\tilde{u}^{i}\right\|_{\infty, 2 \eta}^{2} .
$$

Consequently, for any $i \in \mathcal{I}, u^{i}=\tilde{u}^{i}$ on $[T-2 \eta, T-\eta]$. Thus, for any $s \in[T-2 \eta, T-\eta]$ and $i \in \mathcal{I}$, $Y_{s}^{i, t, x}=\tilde{Y}_{s}^{i, t, x}$. Repeating now this procedure on $[T-3 \eta, T-2 \eta],[T-4 \eta, T-3 \eta]$ etc., we obtain, for any

$i \in \mathcal{I}, u^{i}=\tilde{u}^{i}$. Thus, for any $s \in[t, T]$ and $i \in \mathcal{I}, Y_{s}^{i, t, x}=\tilde{Y}_{s}^{i, t, x}$. Henceforth, $\left(Y^{i, t, x}\right)_{i \in \mathcal{I}}$ is the unique solution to Markovian BSDEs 3.1.

Case 2: We now deal with the general case, i.e., without assuming the boundedness of the functions $f_{i}(t, x, 0,0,0)$ and $h_{i}(x)$. To proceed, let us define, for $s \in[t, T]$

$$
\bar{Y}_{s}^{i}:=Y_{s}^{i} \varphi\left(X_{s}^{t, x}\right) \quad \text { and } \quad \underline{Y}_{s}^{i}:=\tilde{Y}_{s}^{i} \varphi\left(X_{s}^{t, x}\right),
$$

where $\varphi$ is the function defined by (3.15). Next, the same calculations as previously leads to the result of the first case, there exists bounded functions $\left(\bar{u}^{i}\right)_{i \in \mathcal{I}}$ and $\left(\underline{\mathrm{u}}^{i}\right)_{i \in \mathcal{I}}$ such that for any $(t, x) \in[0, T] \times \mathbb{R}^{k}$, and $s \in[t, T], \bar{Y}_{s}^{i}=\bar{u}^{i}\left(s, X_{s}^{t, x}\right)$ and $\underline{Y}_{s}^{i}=\underline{u}^{i}\left(s, X_{s}^{t, x}\right), \forall i \in \mathcal{I}$. Thus, for any $(t, x) \in[0, T] \times \mathbb{R}^{k}$, and $s \in[t, T]$, $\bar{u}^{i}\left(s, X_{s}^{t, x}\right)=\underline{\mathrm{u}}^{i}\left(s, X_{s}^{t, x}\right)$. Then it is enough to take $u^{i}(t, x):=\varphi^{-1}(x) \bar{u}^{i}(t, x)$ and $\tilde{u}^{i}(t, x):=\varphi^{-1}(x) \underline{\mathrm{u}}^{i}(t, x)$, $(t, x) \in[0, T] \times \mathbb{R}^{k}$ and $i \in \mathcal{I}$, which $u^{i}=\tilde{u}^{i}$. Consequently, for any $s \in[t, T], Y_{s}^{i, t, x}=\tilde{Y}_{s}^{i, t, x}$, which means that the solution to Markovian BSDEs (3.1) is unique.

\section{The main result : Existence and uniqueness of the solution for the system of IPDEs with interconnected obstacles}

We now turn to study of the existence and uniqueness in viscosity sense of the solution of the system of integropartial differential equations with interconnected obstacles (2.12). Before doing so, we precise our meaning of the definition of the viscosity solution of this system. It is not exactly the same as in [14] (see also Definition (4.4) in the Appendix).

Definition 4.1 We say that a family of deterministic continuous functions $\vec{u}:=\left(u^{i}\right)_{i \in \mathcal{I}}$ is a viscosity supersolution (resp. subsolution) of (2.12) if: $\forall i \in\{1, \ldots, m\}$,

a) $u^{i}(T, x) \geq($ resp. $\leq) h_{i}(x), \forall x \in \mathbb{R}^{k}$;

b) if $\phi \in \mathcal{C}^{1,2}\left([0, T] \times \mathbb{R}^{k}\right)$ is such that $(t, x) \in[0, T) \times \mathbb{R}^{k}$ a local minimum (resp. maximum) point of $u^{i}-\phi$ 
then

$$
\begin{aligned}
\min \{ & u^{i}(t, x)-\max _{j \in \mathcal{I}^{-i}}\left(u^{j}(t, x)-g_{i j}(t, x)\right) ; \\
& \left.\quad-\partial_{t} \phi(t, x)-\mathcal{L} \phi(t, x)-\tilde{f}_{i}\left(t, x,\left(u^{k}(t, x)\right)_{k=1, \ldots, m},\left(\sigma^{\top} D_{x} \phi\right)(t, x), \mathcal{B}_{i} u^{i}(t, x)\right)\right\} \geq(\text { resp. } \leq) 0 .
\end{aligned}
$$

We say that $\vec{u}:=\left(u^{i}\right)_{i \in \mathcal{I}}$ is a viscosity solution of $(2.12)$ if it is both a supersolution and subsolution of (2.12).

Remark 4.2 In our definition, we have to put $\mathcal{B}_{i} u^{i}(t, x)$ instead $\mathcal{B}_{i} \phi(t, x)$, where $\phi$ is the test function. Indeed, $\mathcal{B}_{i} u^{i}(t, x)$ is well defined since $u^{i}$ has a polynomial growth, $\beta$ is bounded and the measure $\lambda$ is finite.

We are now able to state the main result of this paper. Let $\left(Y^{i, t, x}, Z^{i, t, x}, V^{i, t, x}, K^{i, t, x}\right)_{i \in \mathcal{I}}$ be the solution of (3.1) and let $\left(u^{i}\right)_{i \in \mathcal{I}}$ be the continuous functions with polynomial growth such that for any $(t, x) \in[0, T] \times \mathbb{R}^{k}$, $i \in \mathcal{I}$ and $s \in[t, T]$,

$$
Y_{s}^{i, t, x}=u^{i}\left(s, X_{s}^{t, x}\right)
$$

We then have:

Theorem 4.3 The functions $\left(u^{i}\right)_{i \in \mathcal{I}}$ is the unique viscosity solution of the system (2.12), according to Definition (4.1), in the class of continuous functions of polynomial growth.

Proof: We first show that $\left(u^{i}\right)_{i \in \mathcal{I}}$ is a viscosity solution of system (2.12). So let us consider the following system of reflected BSDEs:

$$
\left\{\begin{aligned}
\underline{\mathbf{Y}}^{i, t, x} \in \mathcal{S}^{2}, \underline{\mathrm{Z}}^{i, t, x} \in \mathcal{H}^{2, d}, \underline{\mathbf{V}}^{i, t, x} \in \mathcal{H}^{2}\left(\mathcal{L}^{2}(\lambda)\right), \text { and } \underline{\mathrm{K}}^{i, t, x} \in \mathcal{A}^{2} ; \\
\underline{\mathbf{Y}}_{s}^{i, t, x}=h_{i}\left(X_{T}^{t, x}\right)+\int_{s}^{T} \tilde{f}_{i}\left(r, X_{r}^{t, x},\left(\underline{\mathrm{Y}}_{r}^{k, t, x}\right)_{k \in \mathcal{I}}, \underline{\mathrm{Z}}_{r}^{i, t, x}, \int_{E} \gamma\left(X_{r}^{t, x}, e\right)\left\{u^{i}\left(r, X_{r^{-}}^{t, x}+\beta\left(X_{r^{-}}^{t, x}, e\right)\right)\right.\right. \\
\left.\left.\quad-u^{i}\left(r, X_{r^{-}}^{t, x}\right)\right\} \lambda(d e)\right) d r+\underline{\mathbf{K}}_{T}^{i, t, x}-\underline{\mathbf{K}}_{s}^{i, t, x}-\int_{s}^{T} \underline{\mathbf{Z}}_{r}^{i, t, x} d B_{r}-\int_{s}^{T} \int_{E} \underline{\mathbf{V}}_{r}^{i, t, x}(e) \tilde{\mu}(d r, d e), \\
\underline{\mathbf{Y}}_{s}^{i, t, x} \geqslant \max _{j \in \mathcal{I}^{-i}}\left(\underline{\mathrm{Y}}_{s}^{j, t, x}-g_{i j}\left(s, X_{s}^{t, x}\right)\right) \text { and } \int_{t}^{T}\left(\underline{\mathrm{Y}}_{s}^{i, t, x}-\max _{j \in \mathcal{I}^{-i}}\left(\underline{\mathrm{Y}}_{s}^{j, t, x}-g_{i j}\left(s, X_{s}^{t, x}\right)\right)\right) d \underline{\mathbf{K}}_{s}^{i, t, x}=0 .
\end{aligned}\right.
$$

As the deterministic functions $\left(u^{i}\right)_{i \in \mathcal{I}}$ are continuous and of polynomial growth, $\beta(x, e)$ and $\gamma_{i}(x, e)$ verify respectively (2.4) and (2.8) and finally by Theorem (3.1), the solution of this system exists and is unique. More precisely, the functions $\left(h_{i}\right)_{i \in \mathcal{I}},\left(g_{i j}\right)_{i, j \in \mathcal{I}}$ and

$$
(t, x, y, z) \longmapsto \tilde{f}_{i}\left(t, x, y, z, \int_{E} \gamma(x, e)\left\{u^{i}(t, x+\beta(x, e))-u^{i}(t, x)\right\} \lambda(d e)\right)
$$

satisfy the Assumptions (H1)-(H3) and (H4) as well. Moreover, again by Theorem (3.1), there exist deterministic continuous functions of polynomial growth $\left(\underline{\mathbf{u}}^{i}\right)_{i \in \mathcal{I}}$, such that: $i \in \mathcal{I}$ and $s \in[t, T]$,

$$
\underline{\mathrm{Y}}_{s}^{i, t, x}=\underline{\mathrm{u}}^{i}\left(s, X_{s}^{t, x}\right) .
$$

Finally, using a result by Hamadène-Zhao [14], we deduce that $\left(\underline{u}^{i}\right)_{i \in \mathcal{I}}$ is a solution in viscosity sense of the following system of IPDE with interconnected obstacle:

$$
\left\{\begin{array}{l}
\min \left\{\underline{\mathbf{u}}^{i}(t, x)-\max _{j \in \mathcal{I}^{-i}}\left(\underline{\mathbf{u}}^{j}(t, x)-g_{i j}(t, x)\right) ;\right. \\
\left.\quad-\partial_{t} \underline{\mathbf{u}}^{i}(t, x)-\mathcal{L} \underline{\mathbf{u}}^{i}(t, x)-\tilde{f}_{i}\left(t, x,\left(\underline{\mathbf{u}}^{k}(t, x)\right)_{k=1, \ldots, m},\left(\sigma^{\top} D_{x} \underline{\mathbf{u}}^{i}\right)(t, x), \mathcal{B}_{i} u^{i}(t, x)\right)\right\}=0 ; \\
\underline{\mathbf{u}}^{i}(T, x)=h_{i}(x),
\end{array}\right.
$$


Let us notice that, in this system (4.2), the last component of $\tilde{f}_{i}$ is $\mathcal{B}_{i} u^{i}(t, x)$ and not $\mathcal{B}_{i} \underline{\underline{u}}(t, x)$. Next, recall that $\left(Y^{i, t, x}, Z^{i, t, x}, V^{i, t, x}, K^{i, t, x}\right)_{i \in \mathcal{I}}$ solves the system of reflected BSDEs with jumps with interconnected obstacles (3.1). Therefore, we now that for any $(t, x) \in[0, T] \times \mathbb{R}^{k}, i \in \mathcal{I}$ and $s \in[t, T]$,

$$
V_{s}^{i, t, x}(e)=u^{i}\left(s, X_{s^{-}}^{t, x}+\beta\left(X_{s^{-}}^{t, x}, e\right)\right)-u^{i}\left(s, X_{s^{-}}^{t, x}\right), d s \otimes d \mathbb{P} \otimes d \lambda \text { on }[t, T] \times \Omega \times E .
$$

Then $\left(Y^{i, t, x}, Z^{i, t, x}, V^{i, t, x}, K^{i, t, x}\right)_{i \in \mathcal{I}}$ verify: for any $s \in[t, T]$ and $i \in \mathcal{I}$,

$$
\left\{\begin{aligned}
& Y_{s}^{i, t, x}= h_{i}\left(X_{T}^{t, x}\right)+\int_{s}^{T} \tilde{f}_{i}\left(r, X_{r}^{t, x},\left(Y_{r}^{k, t, x}\right)_{k \in \mathcal{I}}, Z_{r}^{i, t, x}, \int_{E} \gamma\left(X_{r}^{t, x}, e\right)\left\{u^{i}\left(r, X_{r^{-}}^{t, x}+\beta\left(X_{r^{-}}^{t, x}, e\right)\right)\right.\right. \\
&\left.\left.-u^{i}\left(r, X_{r^{-}}^{t, x}\right)\right\} \lambda(d e)\right) d r+K_{T}^{i, t, x}-K_{s}^{i, t, x}-\int_{s}^{T} Z_{r}^{i, t, x} d B_{r}-\int_{s}^{T} \int_{E} V_{r}^{i, t, x}(e) \tilde{\mu}(d r, d e), \\
& Y_{s}^{i, t, x} \geqslant \max _{j \in \mathcal{I}^{-i}}\left(Y_{s}^{, t, x j}-g_{i j}\left(s, X_{s}^{t, x}\right)\right), \\
& \int_{0}^{T}\left(Y_{s}^{i, t, x}-\max _{j \in \mathcal{I}^{-i}}\left(Y_{s}^{j, t, x}-g_{i j}\left(s, X_{s}^{t, x}\right)\right)\right) d K_{s}^{i, t, x}=0 .
\end{aligned}\right.
$$

Therefore, by uniqueness of the solution of the system 4.1), we deduce that for any $s \in[t, T]$ and $i \in \mathcal{I}$, $\underline{\mathrm{Y}}_{s}^{i, t, x}=Y_{s}^{i, t, x}$. Then, for any $i \in \mathcal{I}, \underline{\mathrm{u}}^{i}=u^{i}$. Consequently, $\left(u^{i}\right)_{i \in \mathcal{I}}$ is a viscosity solution of (2.12) in the sense of Definition 4.11.

Now, let us show that $\left(u^{i}\right)_{i \in \mathcal{I}}$ is the unique solution in the class of continuous functions of polynomial growth. It is based on the uniqueness of the markovian solution to BSDEs.

So let $\left(\bar{u}^{i}\right)_{i \in i \in \mathcal{I}}$ be another continuous with polynomial growth solution of (2.12) in the sense of Definition (4.1), i.e., for any $i \in \mathcal{I}$,

$$
\left\{\begin{array}{l}
\min \left\{\bar{u}^{i}(t, x)-\max _{j \in \mathcal{I}^{-i}}\left(\bar{u}^{j}(t, x)-g_{i j}(t, x)\right) ;\right. \\
\left.\quad-\partial_{t} \bar{u}^{i}(t, x)-\mathcal{L} \bar{u}^{i}(t, x)-\tilde{f}_{i}\left(t, x,\left(\bar{u}^{k}(t, x)\right)_{k=1, \ldots, m},\left(\sigma^{\top} D_{x} \bar{u}^{i}\right)(t, x), \mathcal{B}_{i} \bar{u}^{i}(t, x)\right)\right\}=0 ; \\
\bar{u}^{i}(T, x)=h_{i}(x) .
\end{array}\right.
$$

Next, let us consider the following system of reflected BSDEs:

$$
\left\{\begin{aligned}
\bar{Y}^{i, t, x} \in & \mathcal{S}^{2}, \bar{Z}^{i, t, x} \in \mathcal{H}^{2, d}, \bar{V}^{i, t, x} \in \mathcal{H}^{2}\left(\mathcal{L}^{2}(\lambda)\right), \text { and } \bar{K}^{i, t, x} \in \mathcal{A}^{2} ; \\
\bar{Y}_{s}^{i, t, x}= & h_{i}\left(X_{T}^{t, x}\right)+\int_{s}^{T} \tilde{f}_{i}\left(r, X_{r}^{t, x},\left(\bar{Y}_{r}^{k, t, x}\right)_{k \in \mathcal{I}}, \bar{Z}_{r}^{i, t, x}, \int_{E} \gamma\left(X_{r}^{t, x}, e\right)\left\{\bar{u}^{i}\left(r, X_{r^{-}}^{t, x}+\beta\left(X_{r^{-}}^{t, x}, e\right)\right)\right.\right. \\
& \left.\left.-\bar{u}^{i}\left(r, X_{r^{-}}^{t, x}\right)\right\} \lambda(d e)\right) d r+\bar{K}_{T}^{i, t, x}-\bar{K}_{s}^{i, t, x}-\int_{s}^{T} \bar{Z}_{r}^{i, t, x} d B_{r}-\int_{s}^{T} \int_{E} \bar{V}_{r}^{i, t, x}(e) \tilde{\mu}(d r, d e), \\
\bar{Y}_{s}^{i, t, x} \geqslant & \max _{j \in \mathcal{I}^{-i}}\left(\bar{Y}_{s}^{j, t, x}-g_{i j}\left(s, X_{s}^{t, x}\right)\right) \text { and } \int_{t}^{T}\left(\bar{Y}_{s}^{i, t, x}-\max _{j \in \mathcal{I}^{-i}}\left(\bar{Y}_{s}^{j, t, x}-g_{i j}\left(s, X_{s}^{t, x}\right)\right)\right) d \bar{K}_{s}^{i, t, x}=0 .
\end{aligned}\right.
$$

As for the reflected BSDEs (4.1), the solution of the system (4.5) exists and is unique since the deterministic functions $\left(\bar{u}^{i}\right)_{i \in i \in \mathcal{I}}$ are continuous and of polynomial growth. Moreover, there exists a deterministic continuous functions of polynomial growth $\left(v^{i}\right)_{i \in \mathcal{I}}$, such that:

$$
\forall s \in[t, T], \bar{Y}_{s}^{i, t, x}=v^{i}\left(s, X_{s}^{t, x}\right) .
$$


and

$$
\bar{V}_{s}^{i, t, x}(e)=v^{i}\left(s, X_{s^{-}}^{t, x}+\beta\left(X_{s^{-}}^{t, x}, e\right)\right)-v^{i}\left(s, X_{s^{-}}^{t, x}\right), d s \otimes d \mathbb{P} \otimes d \lambda \text { on }[t, T] \times \Omega \times E .
$$

Then , by using a result by Hamadène-Zhao [14], $\left(v^{i}\right)_{i \in \mathcal{I}}$ is the unique viscosity solution, in the class of continuous functions with polynomial growth, of the following system:

$$
\left\{\begin{array}{l}
\min \left\{v^{i}(t, x)-\max _{j \in \mathcal{I}^{-i}}\left(v^{j}(t, x)-g_{i j}(t, x)\right) ;\right. \\
\left.\quad-\partial_{t} v^{i}(t, x)-\mathcal{L} v^{i}(t, x)-\tilde{f}_{i}\left(t, x,\left(v^{k}(t, x)\right)_{k=1, \ldots, m},\left(\sigma^{\top} D_{x} v^{i}\right)(t, x), \mathcal{B}_{i} \bar{u}^{i}(t, x)\right)\right\}=0 ; \\
v^{i}(T, x)=h_{i}(x),
\end{array}\right.
$$

Now, as the functions $\left(\bar{u}^{i}\right)_{i \in i \in \mathcal{I}}$ solves system (4.7), hence by uniqueness of the solution of this system (4.7) (see [14],Proposition 4.2), for any $i \in \mathcal{I}$ one deduces that $\bar{u}^{i}=v^{i}$. Next, by the characterization of the jumps (4.6), for any $i \in \mathcal{I}$, it holds

$$
\bar{V}_{s}^{i, t, x}(e)=\bar{u}^{i}\left(s, X_{s^{-}}^{t, x}+\beta\left(X_{s^{-}}^{t, x}, e\right)\right)-\bar{u}^{i}\left(s, X_{s^{-}}^{t, x}\right), d s \otimes d \mathbb{P} \otimes d \lambda \text { on }[t, T] \times \Omega \times E .
$$

Going back now to (4.5) and replace the quantity $\bar{u}^{i}\left(s, X_{s^{-}}^{t, x}+\beta\left(X_{s^{-}}^{t, x}, e\right)\right)-\bar{u}^{i}\left(s, X_{s^{-}}^{t, x}\right)$ with $\bar{V}_{s}^{i, t, x}(e)$, it follows that: for any $i \in \mathcal{I}$ and $s \in[t, T]$,

$$
\left\{\begin{aligned}
\bar{Y}_{s}^{i, t, x}= & h_{i}\left(X_{T}^{t, x}\right)+\int_{s}^{T} \tilde{f}_{i}\left(r, X_{r}^{t, x},\left(\bar{Y}_{r}^{k, t, x}\right)_{k \in \mathcal{I}}, \bar{Z}_{r}^{i, t, x}, \int_{E} \gamma\left(X_{r}^{t, x}, e\right) \bar{V}_{r}^{i, t, x}(e) \lambda(d e)\right) d r \\
& +\bar{K}_{T}^{i, t, x}-\bar{K}_{s}^{i, t, x}-\int_{s}^{T} \bar{Z}_{r}^{i, t, x} d B_{r}-\int_{s}^{T} \int_{E} \bar{V}_{r}^{i, t, x}(e) \tilde{\mu}(d r, d e), \\
\bar{Y}_{s}^{i, t, x} \geqslant & \max _{j \in \mathcal{I}^{-i}}\left(\bar{Y}_{s}^{j, t, x}-g_{i j}\left(s, X_{s}^{t, x}\right)\right) \text { and } \int_{t}^{T}\left(\bar{Y}_{s}^{i, t, x}-\max _{j \in \mathcal{I}^{-i}}\left(\bar{Y}_{s}^{j, t, x}-g_{i j}\left(s, X_{s}^{t, x}\right)\right)\right) d \bar{K}_{s}^{i, t, x}=0 .
\end{aligned}\right.
$$

But $\left(Y^{i, t, x}\right)$ solves system (4.9). Then, by the uniqueness result of Proposition (3.4), one deduce that

$$
\forall i \in \mathcal{I}, \bar{Y}^{i, t, x}=Y^{i, t, x} \text {. }
$$

Hence, for any $i \in \mathcal{I}$ and $(t, x) \in[0, T] \times \mathbb{R}^{k}, u^{i}(t, x)=\bar{u}^{i}(t, x)=v^{i}(t, x)$ which means that the solition of (2.12), in the sense of Definition (4.1), is unique in the class of continuous functions with polynomial growth.

\section{Appendix}

In the paper by Hamadène and Zhao [14], the definition of the viscosity solution of the system (2.12), is given as follows.

Definition 4.4 Let $\vec{u}:=\left(u^{i}\right)_{i \in \mathcal{I}}$ be a function of $C\left([0, T] \times \mathbb{R}^{k} ; \mathbb{R}^{m}\right)$.

(i) We say that $\vec{u}$ is a viscosity supersolution (resp. subsolution) of (2.12) if: $\forall i \in\{1, \ldots, m\}$,

a) $u^{i}(T, x) \geq($ resp. $\leq) h_{i}(x), \forall x \in \mathbb{R}^{k}$;

b) if $\phi \in \mathcal{C}^{1,2}\left([0, T] \times \mathbb{R}^{k}\right)$ is such that $(t, x) \in[0, T) \times \mathbb{R}^{k}$ a local minimum (resp. maximum) point of $u^{i}-\phi$ then

$$
\begin{aligned}
\min \{ & u^{i}(t, x)-\max _{j \in \mathcal{I}^{-i}}\left(u^{j}(t, x)-g_{i j}(t, x)\right) ; \\
& \left.\quad-\partial_{t} \phi(t, x)-\mathcal{L} \phi(t, x)-\bar{f}_{i}\left(t, x,\left(u^{k}(t, x)\right)_{k=1, \ldots, m},\left(\sigma^{\top} D_{x} \phi\right)(t, x), \mathcal{B}_{i} \phi(t, x)\right)\right\} \geq(\text { resp. } \leq) 0 .
\end{aligned}
$$

(ii) We say that $\vec{u}:=\left(u^{i}\right)_{i \in \mathcal{I}}$ is a viscosity solution of (2.12) if it is both a supersolution and subsolution of (2.12). 


\section{References}

[1] O. Alvarez and A. Tourin. Viscosity solutions of nonlinear integro-diffrential equations. Ann. Inst. H. Poincaré-Anal. Non Linéaire, 13(3), pp. 293-317, 1996.

[2] G. Barles, R. Buckdahn, and E. Pardoux. Backward stochastic differential equations and integral-partial differential equations. An International Journal of Probability and Stochastic Processes, vol. 60, no. 1-2, pp. 57-83, 1997.

[3] G. Barles, E. Chasseigne, and C. Imbert. On the Dirichlet problem for second-order elliptic integrodifferential equations. India Univ. Math. J., 57(1), pp. 213âĂŞ-246, 2008.

[4] G. Barles, and C. Imbert. Second-order elliptic integro-differential equations: Viscosity solutions' theory revisited. Ann. Inst. H. Poincaré Anal. Non Linéaire 25(3), pp. 567âĂŞ-585, 2008.

[5] I.H. Biswas, E.R. Jakobsen, and K.H. Karlsen. Viscosity solutions for a system of integro-PDEs and connections to optimal switching and control of jump-diffusion processes. Appl. Math. Optim. 62(1), pp. 47-80, 2010.

[6] E. H. Essaky. Reflected backward stochastic differential equation with jumps and RCLL obstacle. Bulletin des sciences mathematiques, 132(8), pp. 690-710, 2008.

[7] J.-F. Chassagneux, R. Elie, and I. Kharroubi. A note on existence and uniqueness for solutions of multidimensional reflected bsdes. Electronic Communications in Probability, 16, pp. 120-128, 2011

[8] T. Fujiwara and H. Kunita. Stochastic differential equations of jump type and Lévy processes in diffeomorphisms group. Journal of mathematics of Kyoto University, 25(1), pp. 71-106, 1985.

[9] S. Hamadène and M.-A. Morlais. Viscosity solutions of systems of PDEs with interconnected obstacles and switching problem. Applied Mathematics \& Optimization, 67(2):163-196, 2013.

[10] S. Hamadène and M.-A. Morlais. Viscosity solutions for second order integro-differential equations without monotonicity condition: the probabilistic approach. Stochastics, 88(4), pp. 632-649, 2016.

[11] S. Hamadène and Y. Ouknine. Reflected backward stochastic differential equation with jumps and random obstacle. Electronic Journal of Probability, 8(2), pp. 1-20, 2003.

[12] S. Hamadène and J. Zhang. Switching problem and related system of reflected backward sdes. Stochastic Processes and their applications, 120(4):403-426, 2010.

[13] S. Hamadène and X. Zhao. Systems of integro-PDEs with interconnected obstacles and multi-modes switching problem driven by Lèvy process. Nonlinear Differential Equations and Applications NoDEA, 22(6), pp. 1607-1660, 2015.

[14] S. Hamadène and X. Zhao. Viscosity solution of system of variational inequalities with interconnected bilateral obstacles of non-local type. Journal of Dynamics and Differential Equations, 2017.

[15] Y. Hu and S. Tang. Multi-dimensional bsde with oblique reflection and optimal switching. Probability Theory and Related Fields, 147(1):89-121, 2010. 
[16] N.L.P. Lundström, K. Nyström, and M. Olofsson. Systems of variational inequalities for non-local operators related to optimal switching problems: existence and uniqueness Manuscripta mathematica 145(3-4), pp. 407-432, 2014.

[17] N.L.P. Lundström, M. Olofsson, and T. Önskog Existence, uniqueness and regularity of solutions to systems of nonlocal obstacle problems related to optimal switching. Journal of Mathematical Analysis and Application, 475(1):13-31, 2019.

[18] P. Protter Stochastic integration and differential equations. Springer-Verlag, vol.21, 1990.

[19] S. Tang and X. Li. Necessary conditions for optimal control of stochastic systems with random jumps. SIAM Journal on Control and Optimization, 32(5): 1447-1475, 1994.

[20] X. Zhu. Backward stochastic viability property with jumps and applications to the comparison theorem for multidimensional BSDEs with jumps. AIP Conference Proceedings, vol. 1200, pp. 438-441, 2010. 